Supporting Information for:

\title{
Using palladium and gold-palladium nanoparticles decorated with molybdenum oxide for versatile hydrogen peroxide electroproduction on graphene nanoribbons
}

Guilherme V. Fortunato $^{a, b^{*}}$, Leticia S. Bezerra ${ }^{b}$, Eduardo S. F. Cardoso ${ }^{b}$, Matheus S. Kronka $^{a}$, Alexsandro J. Santos ${ }^{a}$, Anderson S. Greco ${ }^{c}$, Jorge L. R. Júnior ${ }^{b}$, Marcos R. V. Lanza ${ }^{a}$, Gilberto Maia $^{b^{*}}$

aSão Carlos Institute of Chemistry, University of São Paulo, Avenida Trabalhador São-Carlense 400, São Carlos, SP 13566-590, Brazil

${ }^{b}$ Institute of Chemistry, Universidade Federal de Mato Grosso do Sul; Av. Senador Filinto Muller, 1555; Campo Grande, MS 79074-460, Brazil

${ }^{\mathrm{c}}$ Faculty of Exact Sciences and Technology, Federal University of Grande Dourados, Highway Dourados-Itahum, km 12, Dourados, MS 79804-970, Brazil

\footnotetext{
*Corresponding authors' e-mails:

g.fortunato@usp.br (G.V. Fortunato)

gilberto.maia@ufms.br (G. Maia)
} 
Table S1. Initial (including mass and atomic), EDS, TG metal (mass unburned), and TG and EDS mass and atomic percentages for the GNR/Metal samples.

\begin{tabular}{|c|c|c|c|c|c|c|c|}
\hline & $\begin{array}{c}\text { Initial } \\
\text { mass (mg) }\end{array}$ & $\begin{array}{c}\text { Initial } \\
\text { mass } \\
(\%)\end{array}$ & $\begin{array}{l}\text { Initial } \\
\text { atomic } \\
(\%)\end{array}$ & $\begin{array}{c}\text { EDS } \\
\text { metal } \\
\text { mass }(\%)\end{array}$ & $\begin{array}{c}\text { TG metal } \\
\text { mass (mass } \\
\text { unburned) } \\
(\%)\end{array}$ & $\begin{array}{c}\text { TG and } \\
\text { EDS } \\
\operatorname{mass}(\%)\end{array}$ & $\begin{array}{l}\text { TG and } \\
\text { EDS } \\
\text { atomic } \\
(\%)\end{array}$ \\
\hline GNR (control) & $16.0 \mathrm{C}$ & $100 \mathrm{C}$ & $100 \mathrm{C}$ & $100 \mathrm{C}$ & 0.1 & $100 \mathrm{C}$ & $100 \mathrm{C}$ \\
\hline \multirow{4}{*}{ GNR/AuPdMoOx } & $0.90 \mathrm{Au}$ & $5.2 \mathrm{Au}$ & $0.34 \mathrm{Au}$ & $71.74 \mathrm{Au}$ & \multirow{4}{*}{5.7} & $4.09 \mathrm{Au}$ & $0.26 \mathrm{Au}$ \\
\hline & $0.33 \mathrm{Pd}$ & $1.9 \mathrm{Pd}$ & $0.23 \mathrm{Pd}$ & $21.05 \mathrm{Pd}$ & & $1.20 \mathrm{Pd}$ & $0.14 \mathrm{Pd}$ \\
\hline & 0.1 Mo & $0.6 \mathrm{Mo}$ & $0.08 \mathrm{Mo}$ & 7.21 Mo & & $0.41 \mathrm{Mo}$ & $0.05 \mathrm{Mo}$ \\
\hline & $16.0 \mathrm{C}$ & $92.3 \mathrm{C}$ & $99.35 \mathrm{C}$ & & & $94.3 \mathrm{C}$ & $99.55 \mathrm{C}$ \\
\hline \multirow{3}{*}{ GNR/AuPd } & $0.90 \mathrm{Au}$ & $5.2 \mathrm{Au}$ & $0.34 \mathrm{Au}$ & & \multirow{3}{*}{6.4} & $3.76 \mathrm{Au}$ & $0.24 \mathrm{Au}$ \\
\hline & $0.33 \mathrm{Pd}$ & $1.9 \mathrm{Pd}$ & $0.23 \mathrm{Pd}$ & $58.73 \mathrm{Au}$ & & $2.64 \mathrm{Pd}$ & $0.32 \mathrm{Pd}$ \\
\hline & $16.0 \mathrm{C}$ & $92.9 \mathrm{C}$ & $99.43 \mathrm{C}$ & & & $93.6 \mathrm{C}$ & $99.44 \mathrm{C}$ \\
\hline \multirow{3}{*}{ GNR/AuMoOx } & $0.90 \mathrm{Au}$ & $5.3 \mathrm{Au}$ & $0.34 \mathrm{Au}$ & $90.05 \mathrm{Au}$ & \multirow{3}{*}{4.9} & $4.41 \mathrm{Au}$ & $0.28 \mathrm{Au}$ \\
\hline & $0.1 \mathrm{Mo}$ & $0.6 \mathrm{Mo}$ & $0.08 \mathrm{Mo}$ & $9.95 \mathrm{Mo}$ & & 0.49 Mo & $0.06 \mathrm{Mo}$ \\
\hline & $16.0 \mathrm{C}$ & $94.1 \mathrm{C}$ & $99.58 \mathrm{C}$ & & & $95.1 \mathrm{C}$ & $99.66 \mathrm{C}$ \\
\hline \multirow{3}{*}{ GNR/PdMoOx } & $0.33 \mathrm{Pd}$ & $2.0 \mathrm{Pd}$ & $0.23 \mathrm{Pd}$ & $85.4 \mathrm{Pd}$ & \multirow{3}{*}{2.3} & $1.96 \mathrm{Pd}$ & $0.23 \mathrm{Pd}$ \\
\hline & 0.1 Mo & $0.6 \mathrm{Mo}$ & $0.08 \mathrm{Mo}$ & 14.6 Mo & & $0.34 \mathrm{Mo}$ & $0.04 \mathrm{Mo}$ \\
\hline & $16.0 \mathrm{C}$ & $97.4 \mathrm{C}$ & $99.69 \mathrm{C}$ & & & $97.7 \mathrm{C}$ & $99.73 \mathrm{C}$ \\
\hline \multirow{2}{*}{ GNR/Au } & $0.90 \mathrm{Au}$ & $5.3 \mathrm{Au}$ & $0.34 \mathrm{Au}$ & - & \multirow{2}{*}{5.2} & $5.2 \mathrm{Au}$ & $0.33 \mathrm{Au}$ \\
\hline & $16.0 \mathrm{C}$ & $94.7 \mathrm{C}$ & $99.66 \mathrm{C}$ & & & $94.8 \mathrm{C}$ & $99.67 \mathrm{C}$ \\
\hline \multirow{2}{*}{ GNR/Pd } & $0.33 \mathrm{Pd}$ & $2.0 \mathrm{Pd}$ & $0.23 \mathrm{Pd}$ & - & \multirow{2}{*}{2.0} & $2.0 \mathrm{Pd}$ & $0.23 \mathrm{Pd}$ \\
\hline & $16.0 \mathrm{C}$ & $98.0 \mathrm{C}$ & $99.77 \mathrm{C}$ & & & $98.0 \mathrm{C}$ & $99.77 \mathrm{C}$ \\
\hline \multirow{2}{*}{ GNR/MoOx } & $0.1 \mathrm{Mo}$ & $0.6 \mathrm{Mo}$ & $0.08 \mathrm{Mo}$ & - & \multirow[b]{2}{*}{1.7} & 1.7 Mo & $0.22 \mathrm{Mo}$ \\
\hline & $16.0 \mathrm{C}$ & $99.4 \mathrm{C}$ & $99.92 \mathrm{C}$ & & & $98.3 \mathrm{C}$ & $99.78 \mathrm{C}$ \\
\hline
\end{tabular}




\section{ICP OES Analyses}

The sample preparation was based on a microwave assisted acid digestion procedure using a MARS 6 (CEM Corp., Matthews, NC, USA) microwave system equipped with 8 TFM Teflon ${ }^{\circledR}$ vessels of 110-mL. Aliquots of $6.0 \mathrm{~mL}$ of nitric acid [65\% (v/v), Sigma Aldrich ${ }^{\circledR}$, St. Louis, MO, USA] were added into every carbonaceous sample. The mixtures rested for $12 \mathrm{~h}$ (overnight) under room temperature to start the digestion process. Then, the microwave-assisted digestion procedure was carried out using the following heating program (step - power / ramp time / hold time / temperature): step $1-950 \mathrm{~W} / 20 \mathrm{~min} / 50 \mathrm{~min} / 230{ }^{\circ} \mathrm{C}$; step $2-0 \mathrm{~W} / 0 \mathrm{~min} / 20 \mathrm{~min} / 30^{\circ} \mathrm{C}$ (ventilation). The final digested samples were transferred to a volumetric flask and the volume was made up to $10.0 \mathrm{~mL}, 25.0 \mathrm{~mL}$ (for GNR/PdMoOx) or $250.0 \mathrm{~mL}$ (for GNR/Au) with Milli-Q water. By the way, GNR/AuPdMoOx, GNR/AuMoOx and Vulcan/AuPdMoOx samples solutions were diluted 100x for the determination of $\mathrm{Au}$, and 10x for the determination of Pd. For GNR/AuPd sample solution were diluted 10x for the determination of Au. Blank samples for microwave digestions were prepared by following the procedure described above using acid only.

The determinations of Au, Pd and Mo in GNR-based materials in the sample digests were carried out by a Thermo Scientific iCAP 6300 inductively coupled plasma optical emission spectrometer (ICP OES) (Thermo Fisher Scientific ${ }^{\circledR}$, Bremen, Germany) with a high performance CID86 chip detector. The equipment consists of an EMT-type torch positioned under the concentric glass nebulizer assembly and glass cyclonic spray chamber, with a wavelength range of $166-847 \mathrm{~nm}$ provided by Echelle diffraction grating, with a spectral bandpass range of $7 \mathrm{pm}$ to $200 \mathrm{~nm}$, and a radio frequency (RF) source operating at 27.12 $\mathrm{MHz}$ in the solid state, which allows applying power in the range of 750-1350 W. Argon 5.0 (99.999\% purity, White Martins ${ }^{\circledR}$, Campo Grande, MS, Brazil) was used for plasma generation, nebulization and auxiliary gas. The instrumental parameters used to determine the elements are described in Table S2. Multi-element analytical curves were prepared in $1.0 \%(\mathrm{~m} / \mathrm{v}) \mathrm{HNO}_{3}$ by appropriate dilution of single-element standard stock solutions containing $1000 \mathrm{mg} \mathrm{L}^{-1}$ (SpecSol ${ }^{\circledR}$, SRM-682, USA) of selected elements. The signal intensity response for blank, standard or samples were obtained using three replicates. 
Table S2. Instrumental conditions used to determine $\mathrm{Au}, \mathrm{Pd}$ and Mo by ICP OES.

\begin{tabular}{c|ccc}
\hline & \multicolumn{3}{|c}{ Instrumental parameters } \\
\hline RF applied power & & $1150 \mathrm{~W}$ & \\
Pump speed & & $50 \mathrm{rpm}$ & \\
Plasma gas flow rate & & $12.00 \mathrm{~L} \mathrm{~min}^{-1}$ & \\
Auxiliar gas flow rate & & $0.50 \mathrm{~L} \mathrm{~min}^{-1}$ & \\
Nebulizer gas flow rate & & $0.70 \mathrm{~L} \mathrm{~min}^{-1}$ & $\mathrm{R}$ \\
View & & Axial & 0.9995 \\
\hline Element & $\lambda$ & Linear working range & 0.9990 \\
& $(\mathrm{~nm})$ & $\left.(\mathrm{mg} \mathrm{L})^{-1}\right)$ & 0.9992 \\
\hline Au & 267.595 & $0.05-0.50$ & $1.50-6.00$ \\
Pd & 369.172 & $0.50-5.00$ & \\
\hline
\end{tabular}

Table S3. Results $(n=4)$ for the determination of $\mathrm{Au}, \mathrm{Pd}$ and Mo in GNRs by ICP OES.

\begin{tabular}{|c|c|c|c|c|c|c|c|c|c|}
\hline \multirow{3}{*}{ Nanocomposite } & \multicolumn{9}{|c|}{ Element } \\
\hline & \multicolumn{3}{|c|}{$\mathbf{A u}$} & \multicolumn{3}{|c|}{ Pd } & \multicolumn{3}{|c|}{ Mo } \\
\hline & $\mathrm{mg} \mathrm{g}^{-1}$ & $\%$ & RSD, \% & $\mathrm{mg} \mathrm{g}^{-1}$ & $\%$ & RSD, $\%$ & $\mathrm{mg} \mathrm{g}^{-1}$ & $\%$ & RSD, \% \\
\hline GNR/AuPdMoOx & 40.42 & 4.04 & 3.0 & 11.69 & 1.17 & 4.4 & 4.02 & 0.40 & 5.5 \\
\hline GNR/AuPd & 37.23 & 3.72 & 2.6 & 26.04 & 2.60 & 4.0 & - & - & - \\
\hline GNR/AuMoOx & 43.70 & 4.37 & 3.5 & - & - & - & 4.59 & 0.46 & 4.7 \\
\hline GNR/PdMoOx & - & - & - & 19.18 & 1.92 & 5.1 & 3.24 & 0.32 & 3.9 \\
\hline $\mathrm{GNR} / \mathrm{Au}$ & 50.09 & 5.1 & 1.9 & - & - & - & - & - & - \\
\hline GNR/Pd & - & - & - & 19.49 & 1.95 & 5.3 & - & - & - \\
\hline GNR/MoOx & - & - & - & - & - & - & 16.88 & 1.69 & 2.0 \\
\hline Vulcan/AuPdMoOx & 39.92 & 3.99 & 2.2 & 10.81 & 1.08 & 4.9 & 3.70 & 0.37 & 3.1 \\
\hline
\end{tabular}




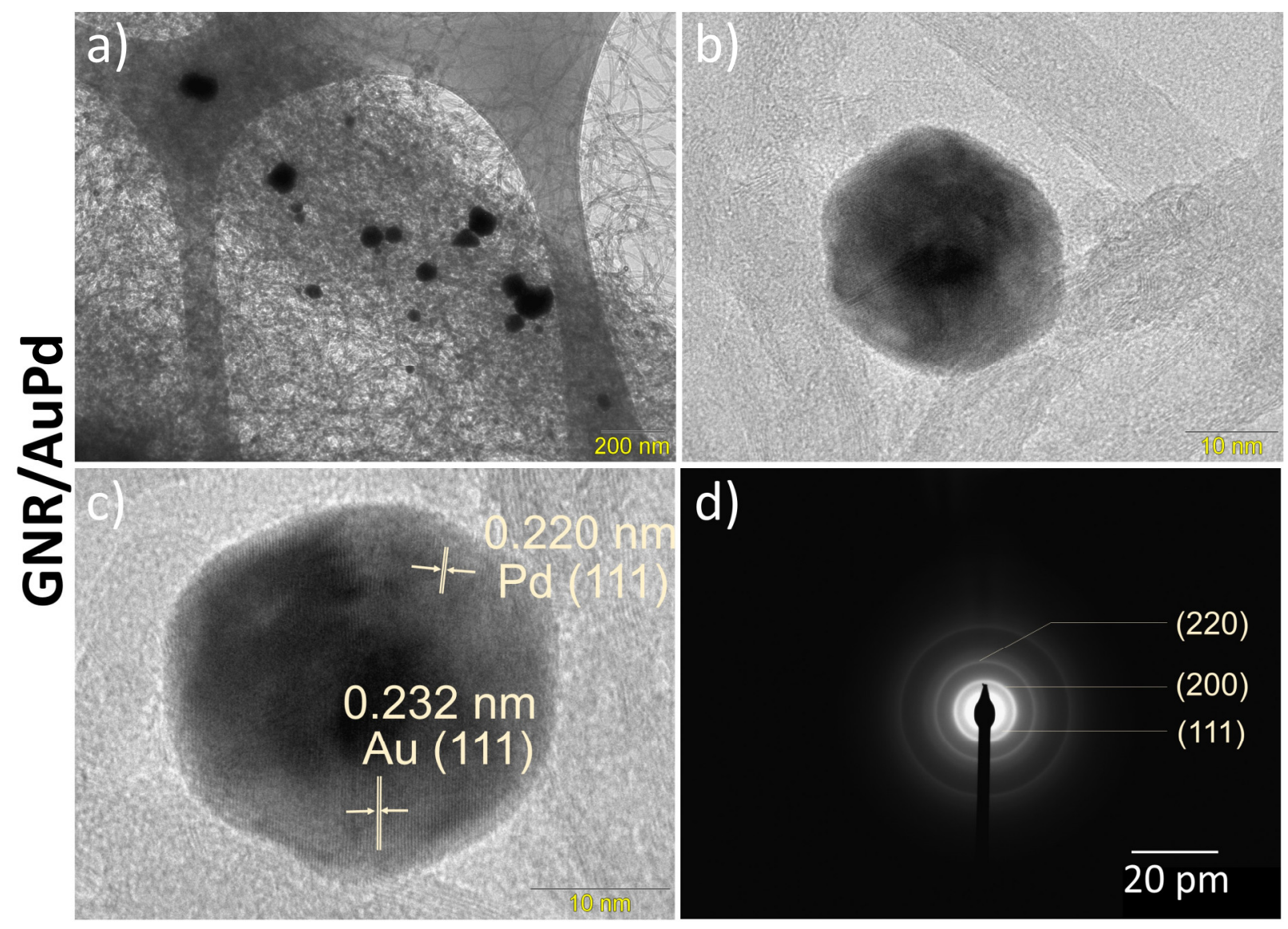

Figure S1. Representative (a) TEM, (b,c) HR-TEM, and (d) electron diffraction pattern images of the GNR/AuPd catalyst. 

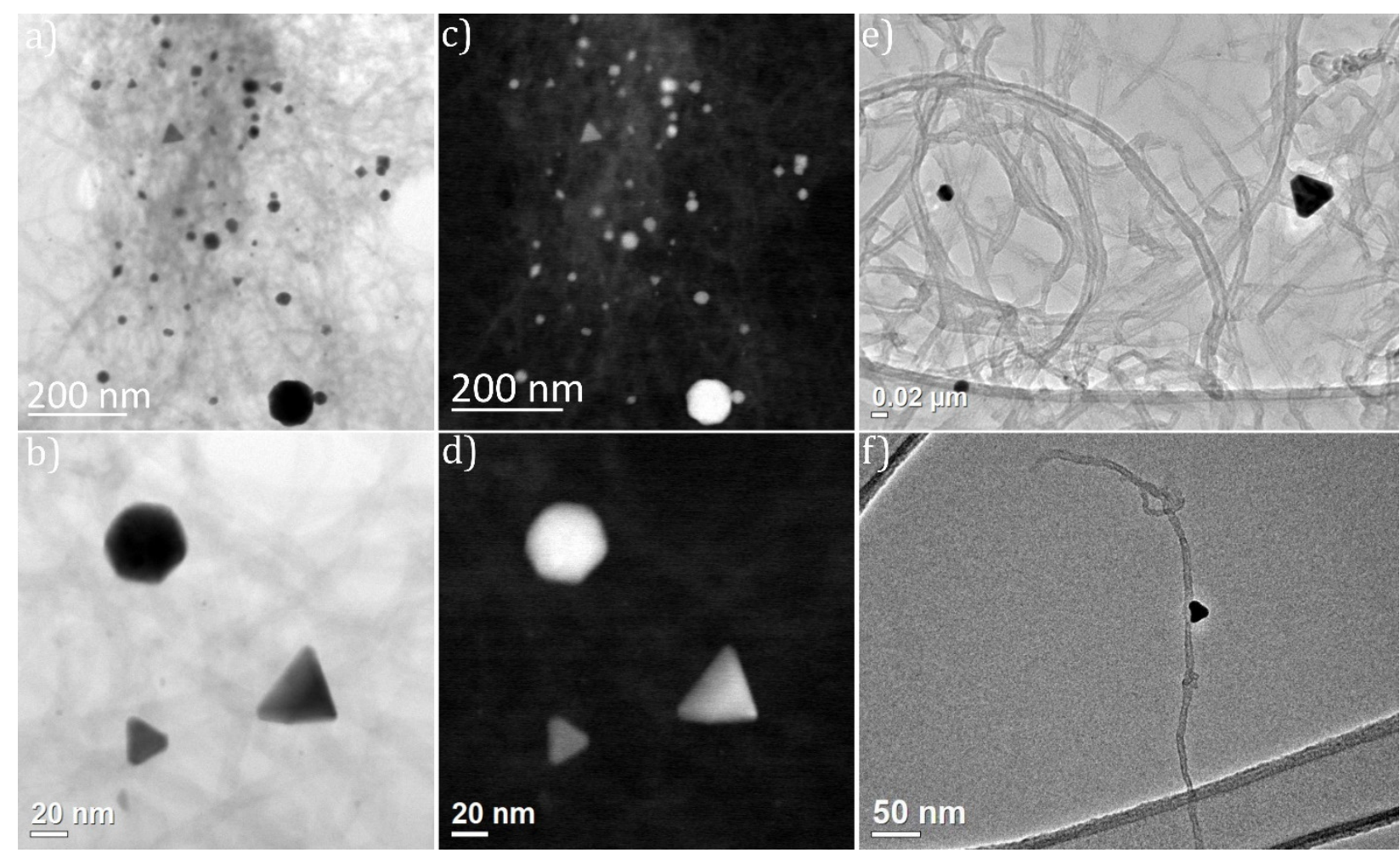

Figure S2. Representative (a,c) BF-, (b,d) DF-STEM, and (e,f) TEM images for the GNR/AuPdMoO ${ }_{x}$ catalyst. 


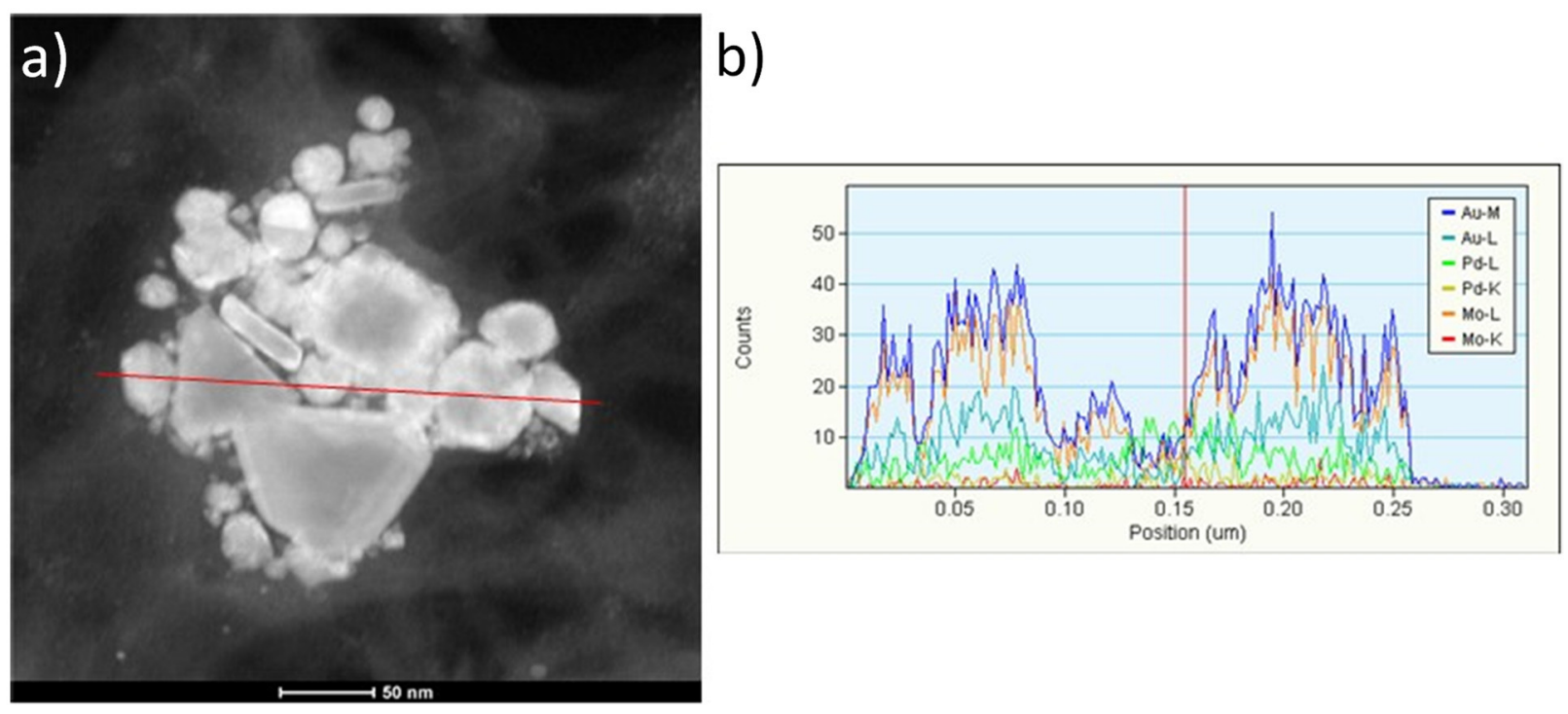

Figure S3. (a) DF-STEM image and (b) EDS line scan analysis of the GNR/AuPdMoO catalyst. 

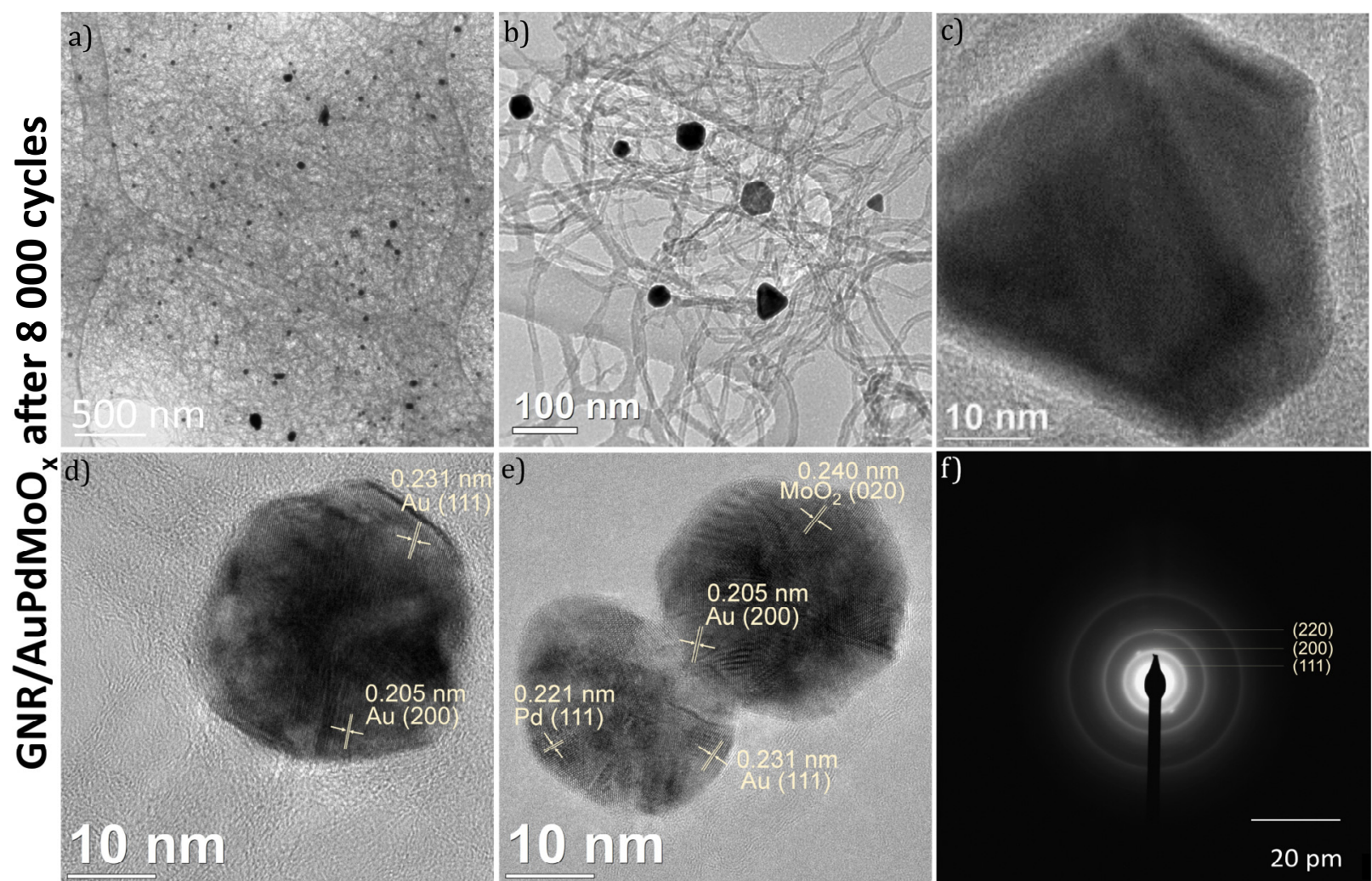

Figure S4. Representative (a,b) TEM, (c-e) HR-TEM, and (f) electron diffraction pattern images of the GNR/AuPdMoO catalyst after stability test. 


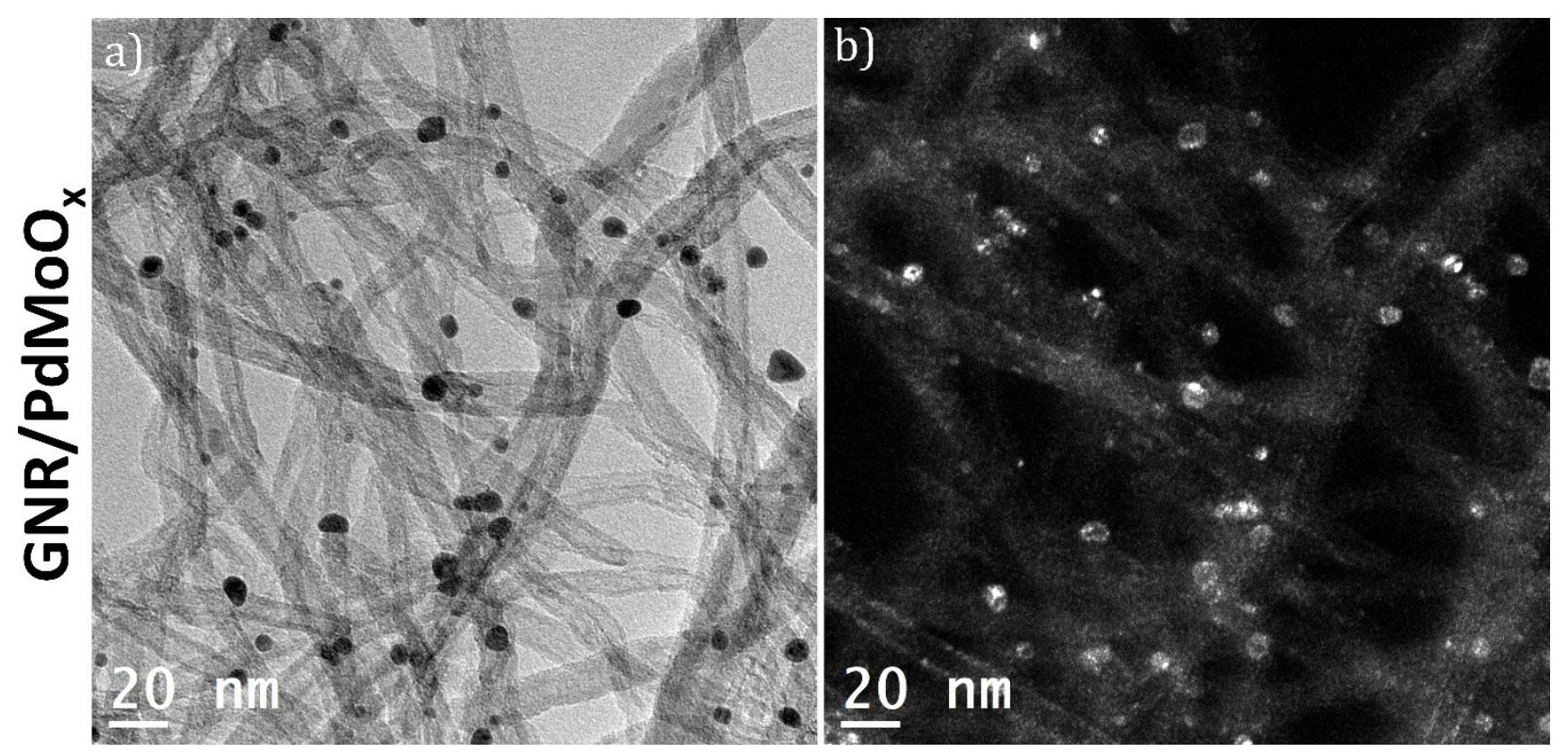

Figure S5. (a) BF- and (b) DF-STEM images for the GNR/PdMoO ${ }_{x}$ catalyst. 

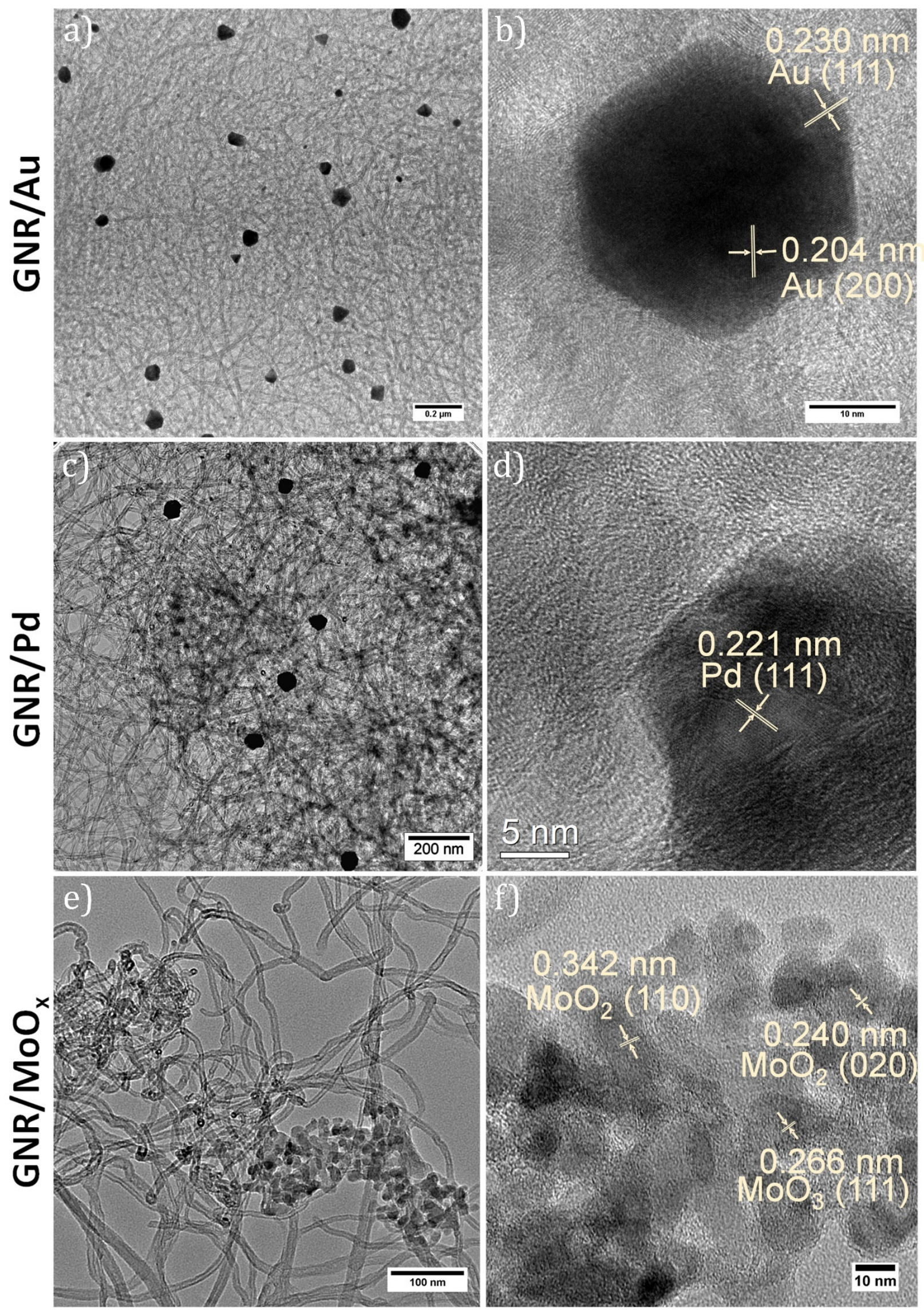

Figure S6. Representative (a,c,e) TEM and (b,d,f) HR-TEM images obtained for the GNR/Au, $\mathrm{GNR} / \mathrm{Pd}$, and $\mathrm{GNR} / \mathrm{MoO}_{\mathrm{x}}$ catalysts. 


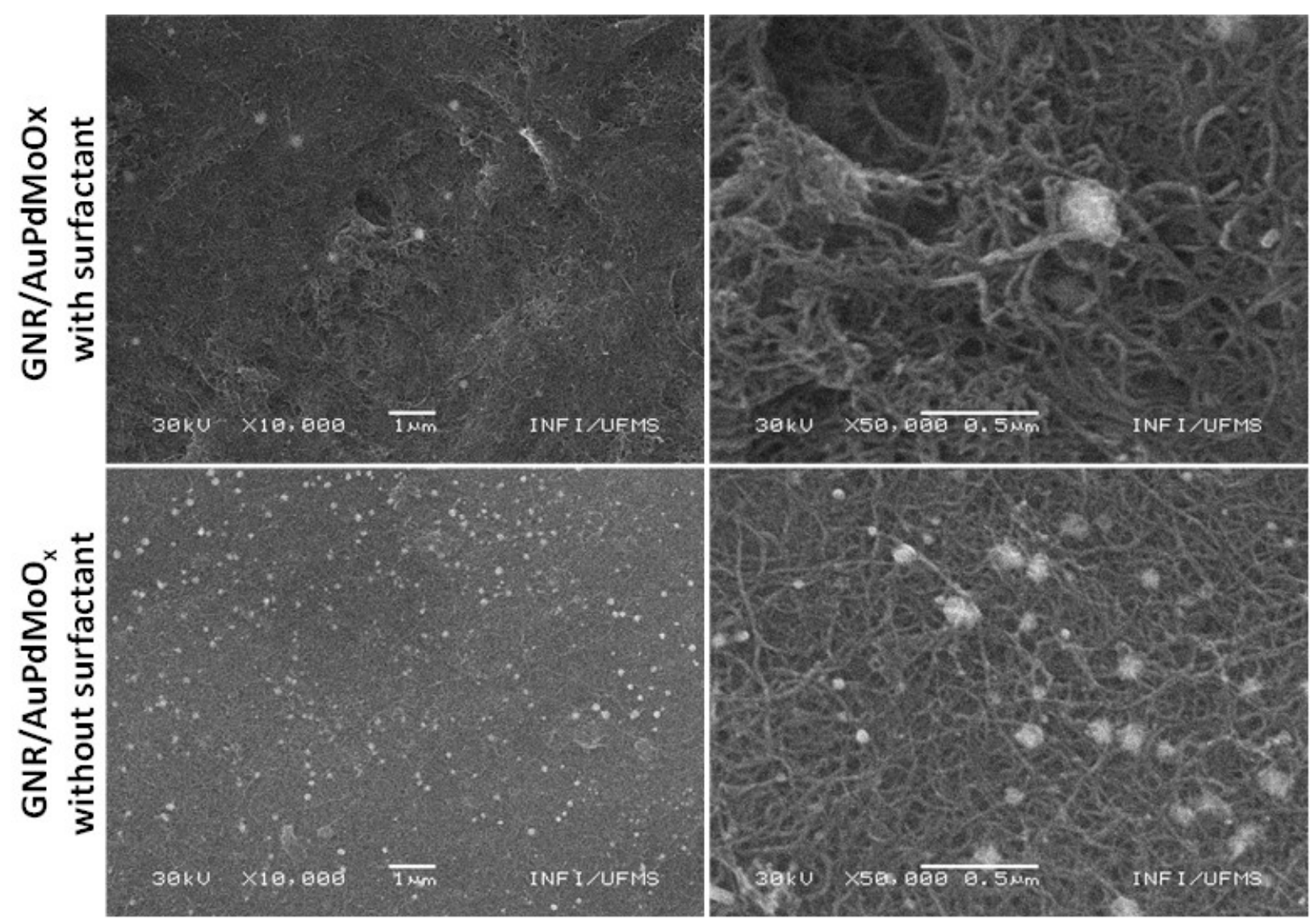

Figure S7. Representative SEM images obtained for the GNR/AuPdMoOx catalyst synthetized with or without the presence of the surfactant Pluronic F-127. 


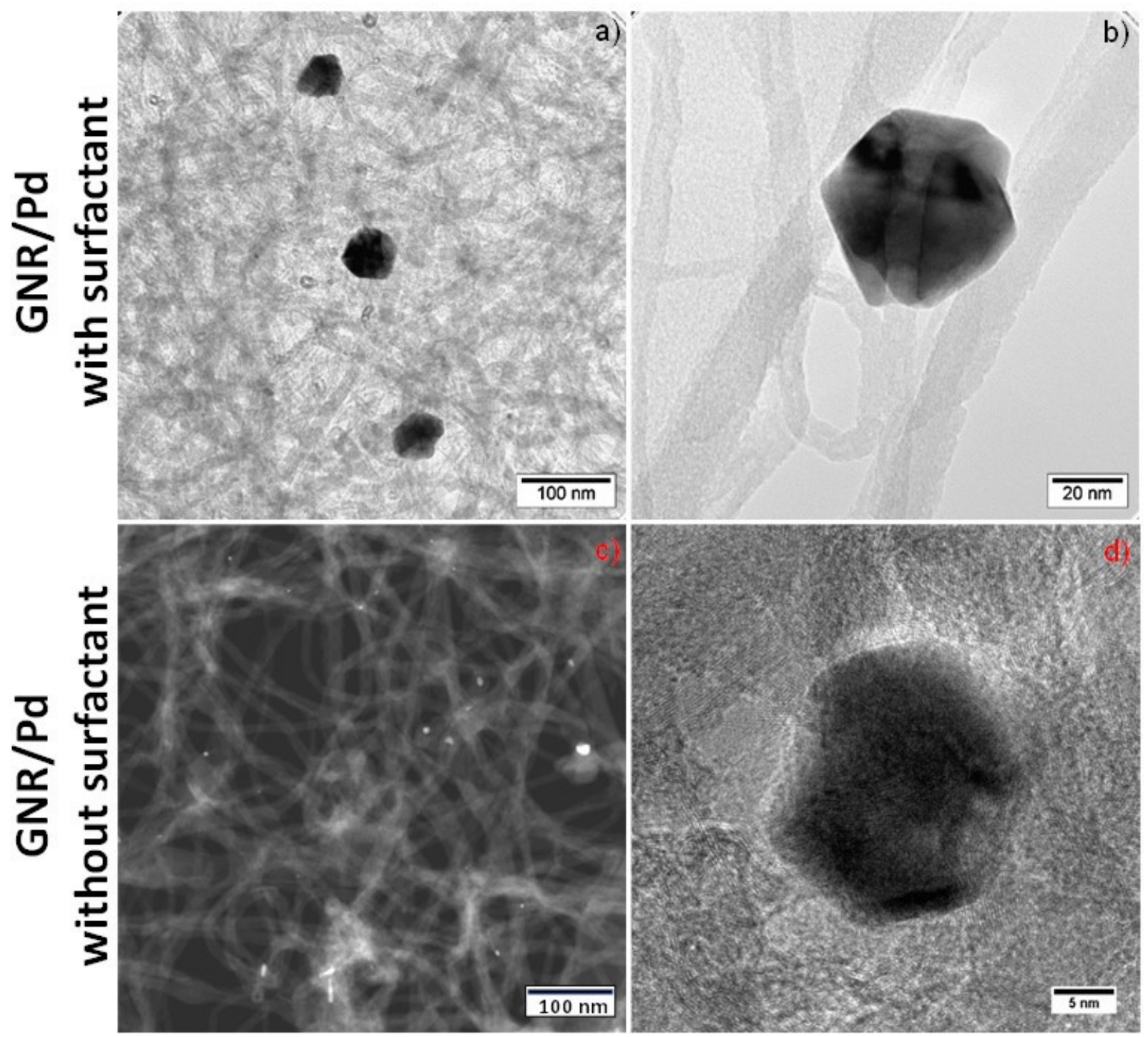

Figure S8. Representative TEM, STEM, and HR-TEM images obtained for the GNR/Pd catalyst synthetized $(a, b)$ with or $(c, d)$ without the presence of the surfactant Pluronic F-127. The Figures (c) and (d) were adapted from de Electronic Supplementary Information of reference ${ }^{1}$. 


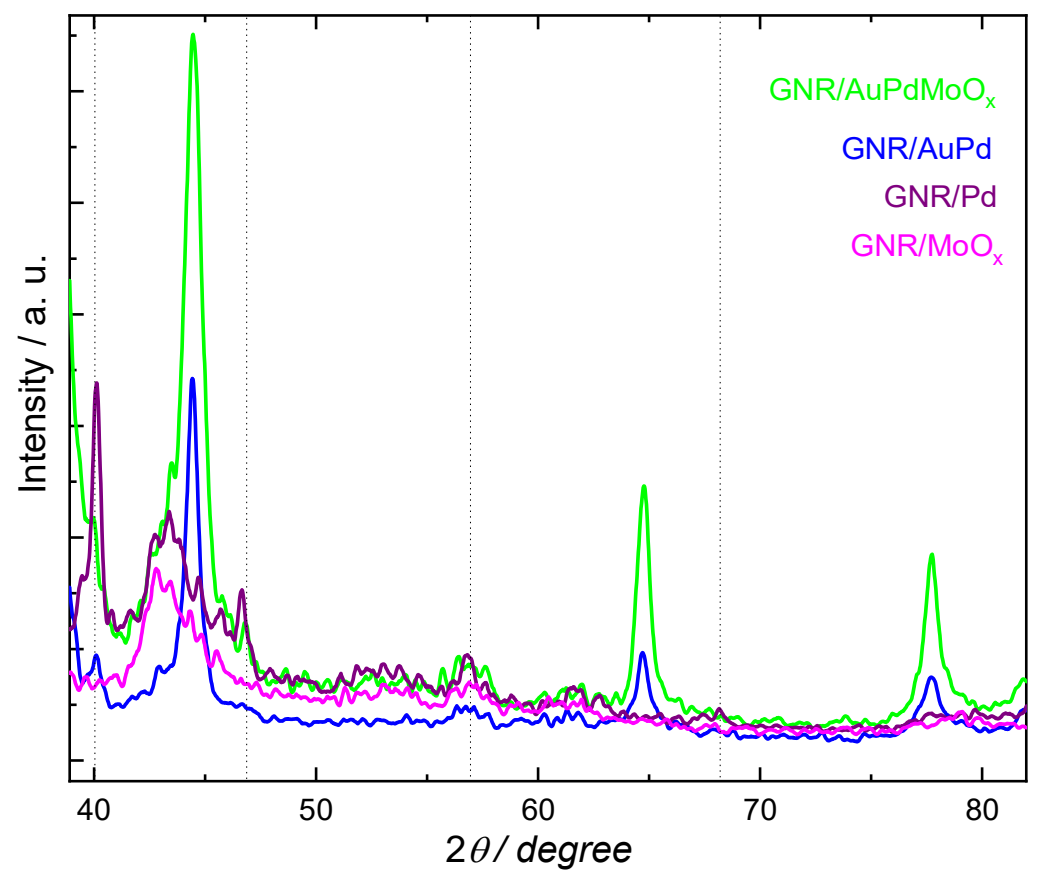

Figure S9. XRD patterns restricted to $2 \theta$ values between $39^{\circ}$ and $82^{\circ}$ obtained for the $\mathrm{GNR} / \mathrm{Au}-\mathrm{Pd} / \mathrm{MoO}_{\mathrm{x}}$, $\mathrm{GNR} / \mathrm{Au}-\mathrm{Pd}, \mathrm{GNR} / \mathrm{Pd}$, and GNR/MoO ${ }_{\mathrm{x}}$ samples. 


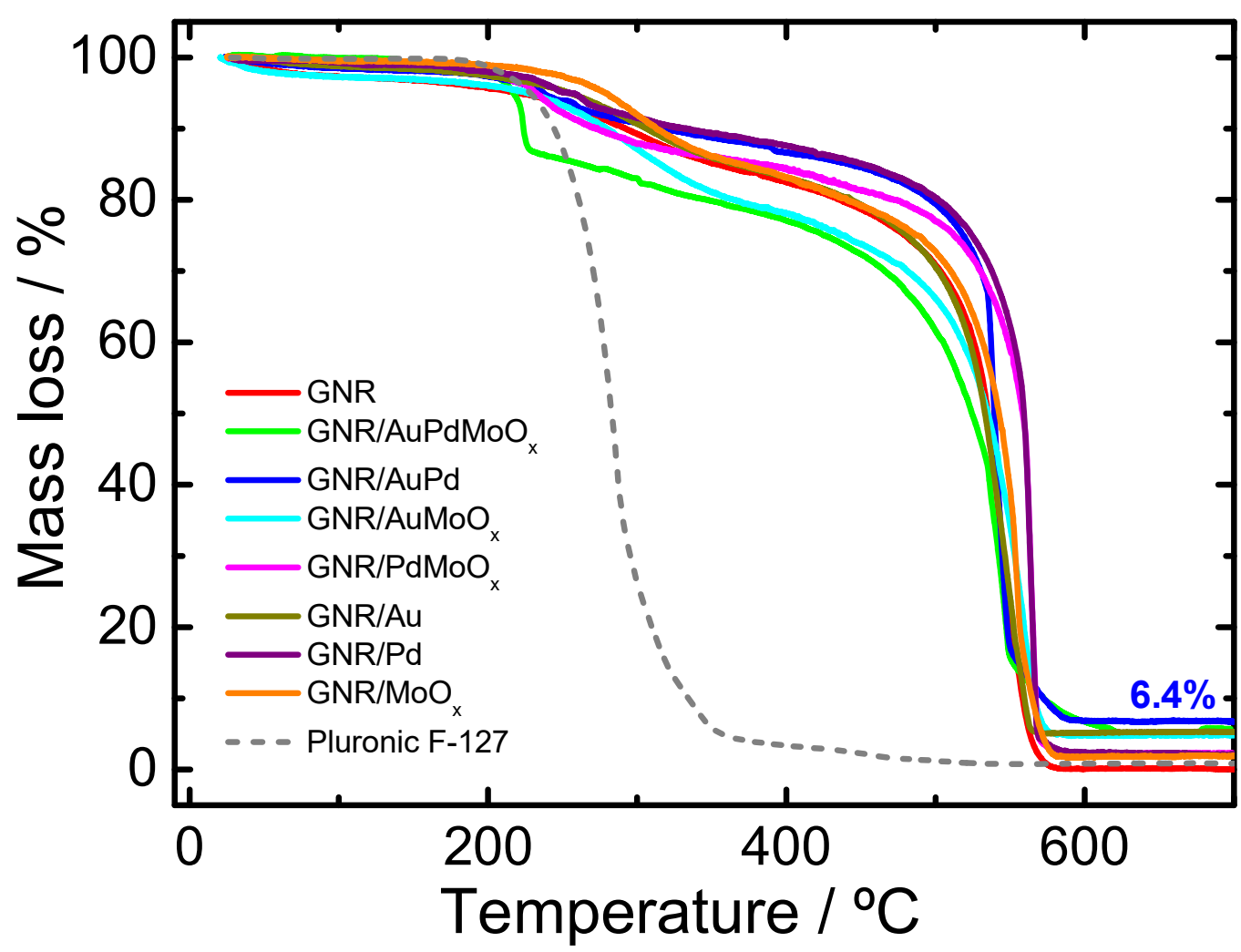

Figure S10. Thermogravimetric responses for the burn of GNR, Pluronic F-127, and GNR/Metals catalysts. 


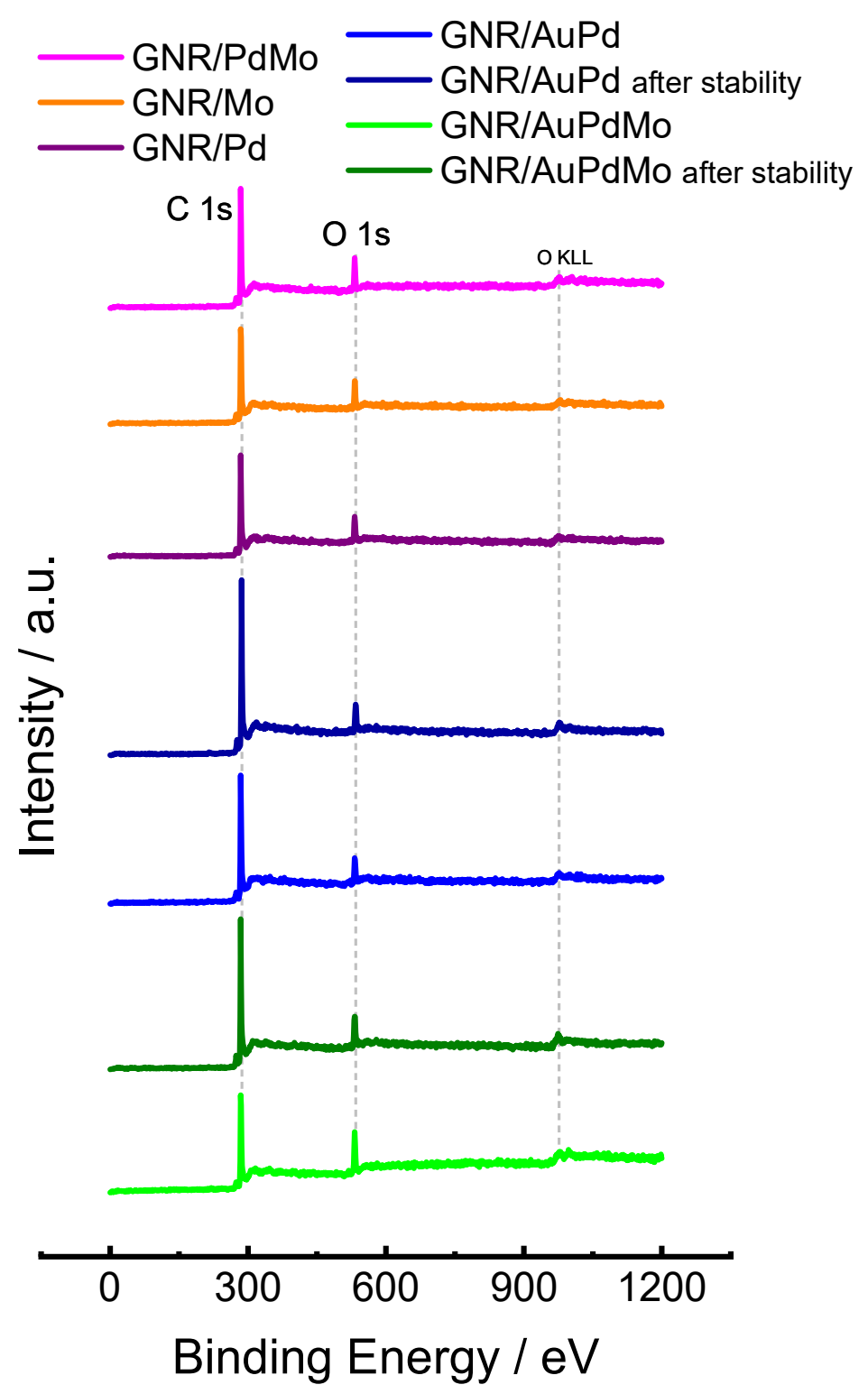

Figure S11. Survey XPS spectra obtained for the GNR/Metal catalysts. 
Table S4. Values related to the peak positions, relative sensitivity factor (R.S.F.), atomic and mass percentages obtained from the XPS spectra shown in Figure S11 for the synthesized catalysts.

\begin{tabular}{|c|c|c|c|c|c|}
\hline Catalyst & Name & $\begin{array}{c}\text { Position } \\
\text { (eV) }\end{array}$ & R.S.F. & $\begin{array}{c}\text { Content } \\
\text { (at. \%) }\end{array}$ & $\begin{array}{c}\text { Content } \\
\text { (mass \%) }\end{array}$ \\
\hline \multirow{2}{*}{ GNR/AuPdMoO } & $\mathrm{O} 1 \mathrm{~s}$ & 532 & 2.93 & 13.78 & 17.56 \\
\hline & $\mathrm{C} 1 \mathrm{~s}$ & 284 & 1 & 86.22 & 82.44 \\
\hline \multirow[t]{2}{*}{ GNR/AuPdMoO ${ }_{x}$ after stability } & $\mathrm{O} 1 \mathrm{~s}$ & 532 & 2.93 & 10.08 & 12.99 \\
\hline & $\mathrm{C} 1 \mathrm{~s}$ & 284 & 1 & 89.92 & 87.01 \\
\hline \multirow[t]{2}{*}{ GNR/AuPd } & $\mathrm{O} 1 \mathrm{~s}$ & 532 & 2.93 & 8.28 & 10.74 \\
\hline & $\mathrm{C} 1 \mathrm{~s}$ & 284 & 1 & 91.72 & 89.26 \\
\hline \multirow[t]{2}{*}{ GNR/AuPd after stability } & $\mathrm{O} 1 \mathrm{~s}$ & 532 & 2.93 & 7.67 & 9.96 \\
\hline & $\mathrm{C} 1 \mathrm{~s}$ & 284 & 1 & 92.33 & 90.04 \\
\hline \multirow[t]{2}{*}{ GNR/PdMoO ${ }_{x}$} & $\mathrm{O} 1 \mathrm{~s}$ & 532 & 2.93 & 10.56 & 13.59 \\
\hline & $\mathrm{C} 1 \mathrm{~s}$ & 284 & 1 & 89.44 & 86.41 \\
\hline \multirow[t]{2}{*}{ GNR/Pd } & $\mathrm{O} 1 \mathrm{~s}$ & 532 & 2.93 & 9.22 & 11.92 \\
\hline & $\mathrm{C} 1 \mathrm{~s}$ & 284 & 1 & 90.78 & 88.08 \\
\hline \multirow[t]{2}{*}{ GNR/MoOx } & $\mathrm{O} 1 \mathrm{~s}$ & 532 & 2.93 & 10 & 12.9 \\
\hline & $\mathrm{C} 1 \mathrm{~s}$ & 284 & 1 & 90 & 87.1 \\
\hline
\end{tabular}


GNR/AuPdMoO
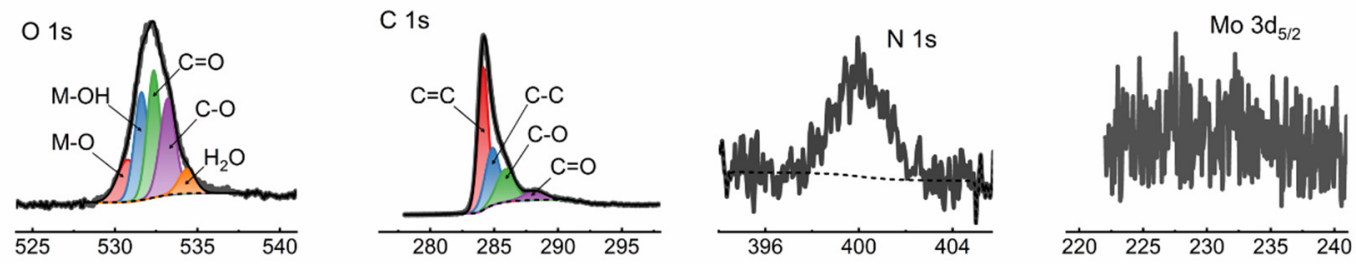

GNR/AuPdMoO $x$ after stability
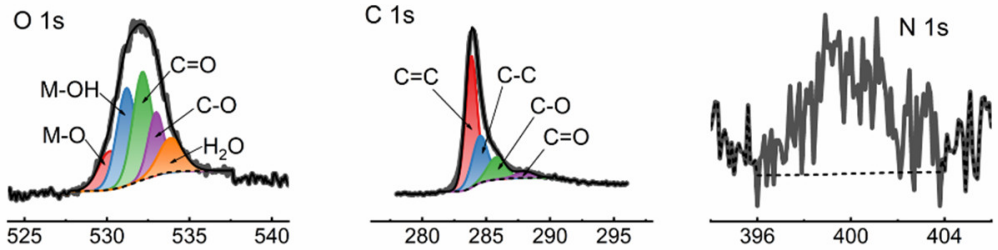

\section{GNR/AuPd}
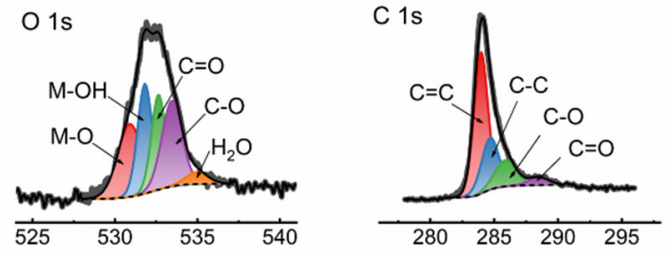

GNR/AuPd after stability
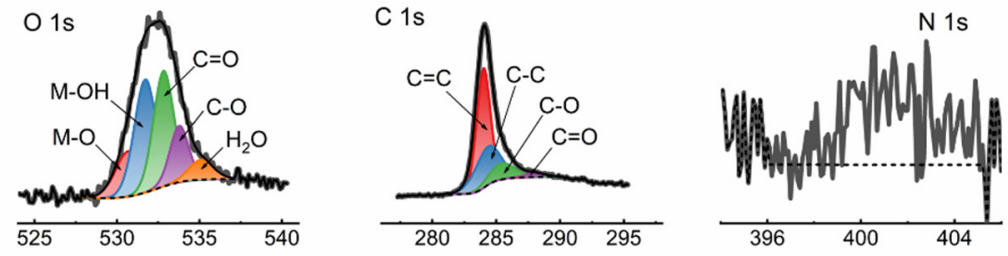

GNR/PdMoO $x$
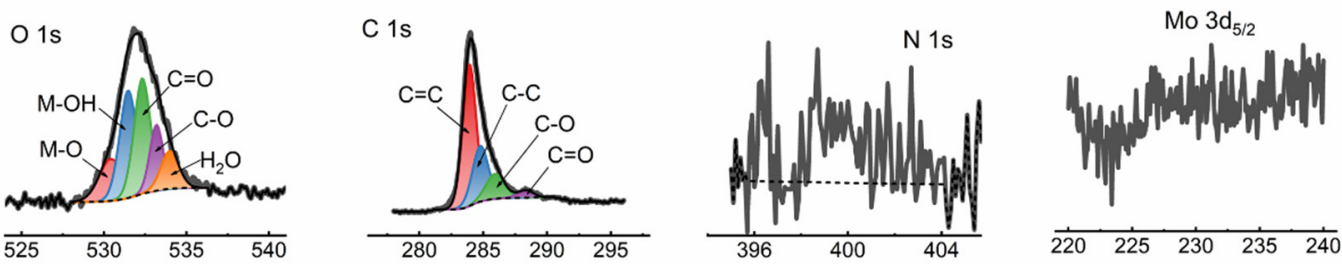

\section{GNR/Pd}
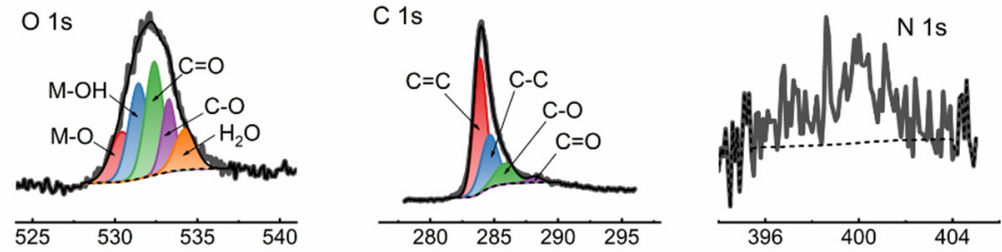

$\mathrm{GNR} / \mathrm{MoO}_{\mathrm{x}}$
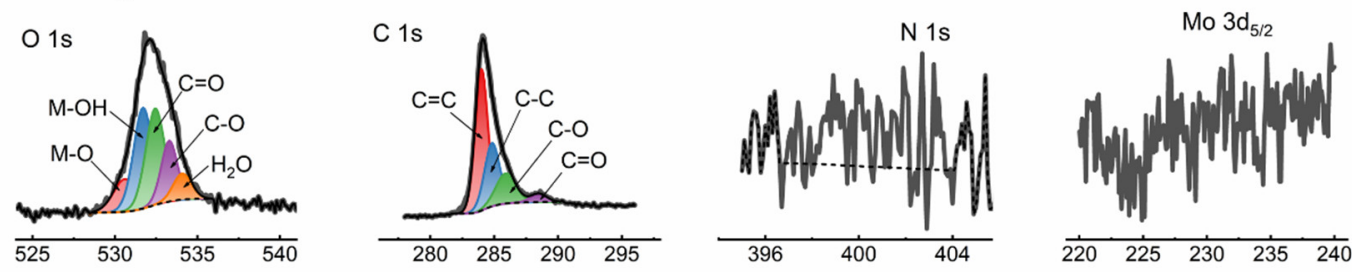

Figure S12. Narrow-scan XPS spectra of O 1s, C 1s, N 1s, and Mo 3d regions obtained for the GNR/Metal catalysts. 
Table S5. Values related to potentials positions and content percentages of the functional groups present in the nanocomposites obtained from high-resolution XPS spectra shown in Figures 4 and S12.

\begin{tabular}{|c|c|c|c|c|}
\hline Catalyst & Name & Group & Position (eV) & $\%$ content \\
\hline \multirow[t]{15}{*}{ GNR/AuPdMoOx } & \multirow[t]{4}{*}{$\operatorname{Pd} 3 d$} & $\mathrm{Pd}^{0} 3 \mathrm{~d}_{5 / 2}$ & 335.34 & 29.62 \\
\hline & & $\mathrm{Pd}^{2+} 3 \mathrm{~d}_{5 / 2}$ & 337.79 & 30.43 \\
\hline & & $\mathrm{Pd}^{0} 3 \mathrm{~d}_{3 / 2}$ & 340.56 & 19.71 \\
\hline & & $\mathrm{Pd}^{2+} 3 \mathrm{~d}_{3 / 2}$ & 342.93 & 20.24 \\
\hline & \multirow{2}{*}{$\mathrm{Au} 4 \mathrm{f}$} & $\mathrm{Au}^{0} 4 \mathrm{f}_{7 / 2}$ & 83.64 & 56.97 \\
\hline & & $A u^{0} 4 f_{5 / 2}$ & 87.27 & 43.03 \\
\hline & \multirow[t]{4}{*}{$\mathrm{C} 1 \mathrm{~s}$} & $\mathrm{C}=\mathrm{C}$ & 284.14 & 46.95 \\
\hline & & $\mathrm{C}-\mathrm{C}$ & 284.87 & 27.62 \\
\hline & & $\mathrm{C}-\mathrm{O}$ & 285.88 & 19.1 \\
\hline & & $\mathrm{C}=\mathrm{O}$ & 288.18 & 6.32 \\
\hline & \multirow[t]{5}{*}{$\mathrm{O} 1 \mathrm{~s}$} & $\mathrm{M}-\mathrm{O}$ & 530.77 & 14.72 \\
\hline & & $\mathrm{M}-\mathrm{OH}$ & 531.6 & 25.53 \\
\hline & & $\mathrm{C}=\mathrm{O}$ & 532.35 & 28.11 \\
\hline & & $\mathrm{C}-\mathrm{O}$ & 533.2 & 24.94 \\
\hline & & $\mathrm{H}_{2} \mathrm{O}$ & 534.36 & 6.7 \\
\hline \multirow{13}{*}{$\begin{array}{l}\text { GNR/AuPdMoO } \\
\text { after stability }\end{array}$} & \multirow{4}{*}{$\operatorname{Pd} 3 d$} & $\mathrm{Pd}^{0} 3 \mathrm{~d}_{5 / 2}$ & 335.07 & 22.19 \\
\hline & & $\mathrm{Pd}^{2+} 3 \mathrm{~d}_{5 / 2}$ & 337.41 & 37.83 \\
\hline & & $\mathrm{Pd}^{0} 3 \mathrm{~d}_{3 / 2}$ & 340.71 & 14.78 \\
\hline & & $\mathrm{Pd}^{2+} 3 \mathrm{~d}_{3 / 2}$ & 343.58 & 25.19 \\
\hline & \multirow[t]{4}{*}{ C 1s } & $\mathrm{C}=\mathrm{C}$ & 283.84 & 54.85 \\
\hline & & $\mathrm{C}-\mathrm{C}$ & 284.52 & 26.5 \\
\hline & & $\mathrm{C}-\mathrm{O}$ & 285.79 & 14.4 \\
\hline & & $\mathrm{C}=\mathrm{O}$ & 288.03 & 4.26 \\
\hline & \multirow[t]{5}{*}{$\mathrm{O} 1 \mathrm{~s}$} & $\mathrm{M}-\mathrm{O}$ & 530.17 & 13.02 \\
\hline & & $\mathrm{M}-\mathrm{OH}$ & 531.18 & 28.4 \\
\hline & & $\mathrm{C}=\mathrm{O}$ & 532.15 & 31.13 \\
\hline & & $\mathrm{C}-\mathrm{O}$ & 532.97 & 15.1 \\
\hline & & $\mathrm{H}_{2} \mathrm{O}$ & 533.86 & 12.35 \\
\hline \multirow[t]{15}{*}{ GNR/AuPd } & \multirow{4}{*}{$\operatorname{Pd} 3 d$} & $\mathrm{Pd}^{0} 3 \mathrm{~d}_{5 / 2}$ & 333.82 & 20.48 \\
\hline & & $\mathrm{Pd}^{2+} 3 \mathrm{~d}_{5 / 2}$ & 337.36 & 39.55 \\
\hline & & $\mathrm{Pd}^{0} 3 \mathrm{~d}_{3 / 2}$ & 340.28 & 13.64 \\
\hline & & $\mathrm{Pd}^{2+} 3 \mathrm{~d}_{3 / 2}$ & 343.17 & 26.34 \\
\hline & \multirow[t]{2}{*}{$\mathrm{Au} 4 \mathrm{f}$} & $\mathrm{Au}^{0} 4 \mathrm{f}_{7 / 2}$ & 83.38 & 57.80 \\
\hline & & $\mathrm{Au}^{0} 4 \mathrm{f}_{5 / 2}$ & 87.04 & 42.20 \\
\hline & \multirow[t]{4}{*}{$\mathrm{C} 1 \mathrm{~s}$} & $\mathrm{C}=\mathrm{C}$ & 283.96 & 54.84 \\
\hline & & $\mathrm{C}-\mathrm{C}$ & 284.68 & 25.36 \\
\hline & & $\mathrm{C}-\mathrm{O}$ & 285.87 & 15.48 \\
\hline & & $\mathrm{C}=\mathrm{O}$ & 288.48 & 4.32 \\
\hline & \multirow[t]{5}{*}{$\mathrm{O} 1 \mathrm{~s}$} & $\mathrm{M}-\mathrm{O}$ & 530.89 & 26.15 \\
\hline & & $\mathrm{M}-\mathrm{OH}$ & 531.81 & 23.96 \\
\hline & & $\mathrm{C}=\mathrm{O}$ & 532.63 & 20.25 \\
\hline & & $\mathrm{C}-\mathrm{O}$ & 533.44 & 25.72 \\
\hline & & $\mathrm{H}_{2} \mathrm{O}$ & 534.96 & 3.92 \\
\hline \multirow{12}{*}{$\begin{array}{c}\text { GNR/AuPd } \\
\text { after stability }\end{array}$} & \multirow[t]{4}{*}{$\operatorname{Pd} 3 d$} & $\mathrm{Pd}^{0} 3 \mathrm{~d}_{5 / 2}$ & 335.72 & 21.9 \\
\hline & & $\mathrm{Pd}^{2+} 3 \mathrm{~d}_{5 / 2}$ & 338.06 & 38.12 \\
\hline & & $\mathrm{Pd}^{0} 3 \mathrm{~d}_{3 / 2}$ & 339.36 & 14.59 \\
\hline & & $\mathrm{Pd}^{2+} 3 \mathrm{~d}_{3 / 2}$ & 342.1 & 25.39 \\
\hline & \multirow[t]{4}{*}{$\mathrm{C} 1 \mathrm{~s}$} & $\mathrm{C}=\mathrm{C}$ & 284.01 & 52.48 \\
\hline & & $\mathrm{C}-\mathrm{C}$ & 284.36 & 31.7 \\
\hline & & $\mathrm{C}-\mathrm{O}$ & 285.47 & 13.26 \\
\hline & & $\mathrm{C}=\mathrm{O}$ & 288.03 & 2.55 \\
\hline & \multirow[t]{4}{*}{$\mathrm{O} 1 \mathrm{~s}$} & $\mathrm{M}-\mathrm{O}$ & 530.71 & 15.9 \\
\hline & & $\mathrm{M}-\mathrm{OH}$ & 531.71 & 32.73 \\
\hline & & $\mathrm{C}=\mathrm{O}$ & 532.82 & 30.99 \\
\hline & & $\mathrm{C}-\mathrm{O}$ & 533.78 & 13.96 \\
\hline
\end{tabular}




\begin{tabular}{|c|c|c|c|c|}
\hline \multirow{14}{*}{$\mathrm{GNR} / \mathrm{PdMoO}_{\mathrm{x}}$} & & $\mathrm{H}_{2} \mathrm{O}$ & 535.09 & 6.42 \\
\hline & \multirow{4}{*}{$\operatorname{Pd} 3 d$} & $\mathrm{Pd}^{0} 3 \mathrm{~d}_{5 / 2}$ & 335.35 & 29.41 \\
\hline & & $\mathrm{Pd}^{2+} 3 \mathrm{~d}_{5 / 2}$ & 337.6 & 30.61 \\
\hline & & $\operatorname{Pd}^{0} 3 \mathrm{~d}_{3 / 2}$ & 339.66 & 19.59 \\
\hline & & $\mathrm{Pd}^{2+} 3 \mathrm{~d}_{3 / 2}$ & 342.35 & 20.39 \\
\hline & \multirow[t]{4}{*}{$\mathrm{C} 1 \mathrm{~s}$} & $\mathrm{C}=\mathrm{C}$ & 283.92 & 56.44 \\
\hline & & C-C & 284.77 & 26.55 \\
\hline & & $\mathrm{C}-\mathrm{O}$ & 285.91 & 13.69 \\
\hline & & $\mathrm{C}=\mathrm{O}$ & 288.32 & 3.31 \\
\hline & \multirow{5}{*}{$\mathrm{O} 1 \mathrm{~s}$} & $\mathrm{M}-\mathrm{O}$ & 530.41 & 15.68 \\
\hline & & $\mathrm{M}-\mathrm{OH}$ & 531.46 & 29.27 \\
\hline & & $\mathrm{C}=\mathrm{O}$ & 532.32 & 30.22 \\
\hline & & $\mathrm{C}-\mathrm{O}$ & 533.18 & 14.07 \\
\hline & & $\mathrm{H}_{2} \mathrm{O}$ & 533.98 & 10.75 \\
\hline \multirow{13}{*}{ GNR/Pd } & \multirow{4}{*}{$\operatorname{Pd} 3 d$} & $\mathrm{Pd}^{0} 3 \mathrm{~d}_{5 / 2}$ & 334.96 & 17.98 \\
\hline & & $\mathrm{Pd}^{2+} 3 \mathrm{~d}_{5 / 2}$ & 337.71 & 42.04 \\
\hline & & $\mathrm{Pd}^{0} 3 \mathrm{~d}_{3 / 2}$ & 341.68 & 11.98 \\
\hline & & $\mathrm{Pd}^{2+} 3 \mathrm{~d}_{3 / 2}$ & 342.84 & 28 \\
\hline & \multirow[t]{4}{*}{$\mathrm{C} 1 \mathrm{~s}$} & $\mathrm{C}=\mathrm{C}$ & 283.88 & 54.54 \\
\hline & & $\mathrm{C}-\mathrm{C}$ & 284.64 & 29.64 \\
\hline & & $\mathrm{C}-\mathrm{O}$ & 285.82 & 13.44 \\
\hline & & $\mathrm{C}=\mathrm{O}$ & 288.21 & 2.39 \\
\hline & \multirow[t]{5}{*}{$\mathrm{O} 1 \mathrm{~s}$} & $\mathrm{M}-\mathrm{O}$ & 530.42 & 16.77 \\
\hline & & $\mathrm{M}-\mathrm{OH}$ & 531.42 & 26.93 \\
\hline & & $\mathrm{C}=\mathrm{O}$ & 532.37 & 29.55 \\
\hline & & $\mathrm{C}-\mathrm{O}$ & 533.26 & 14.44 \\
\hline & & $\mathrm{H}_{2} \mathrm{O}$ & 534.25 & 12.31 \\
\hline \multirow{9}{*}{ GNR/MoO $_{\mathbf{x}}$} & \multirow[t]{4}{*}{$\mathrm{C} 1 \mathrm{~s}$} & $\mathrm{C}=\mathrm{C}$ & 284.01 & 51.01 \\
\hline & & $\mathrm{C}-\mathrm{C}$ & 284.83 & 27.94 \\
\hline & & $\mathrm{C}-\mathrm{O}$ & 285.89 & 17.18 \\
\hline & & $\mathrm{C}=\mathrm{O}$ & 288.48 & 3.86 \\
\hline & \multirow[t]{5}{*}{$\mathrm{O} 1 \mathrm{~s}$} & $\mathrm{M}-\mathrm{O}$ & 530.59 & 12.51 \\
\hline & & $\mathrm{M}-\mathrm{OH}$ & 531.69 & 32.21 \\
\hline & & $\mathrm{C}=\mathrm{O}$ & 532.43 & 30.78 \\
\hline & & $\mathrm{C}-\mathrm{O}$ & 533.29 & 16.54 \\
\hline & & $\mathrm{H}_{2} \mathrm{O}$ & 534.07 & 7.96 \\
\hline
\end{tabular}

The O 1s HR-XPS spectra exhibited a broad peak that was deconvoluted in five peaks for the catalysts investigated in this study (Figure S12 and Table S3). It is worth noting that, with regard to the $\mathrm{M}-\mathrm{O}$ and $\mathrm{M}-\mathrm{OH}$ peaks (where $\mathrm{M}=$ metal), the $\%$ content of $\mathrm{M}-\mathrm{O}$ was found to be relatively lower (about half of the value, on average) than the $\%$ content of $\mathrm{M}-\mathrm{OH}$ in the nanocomposite catalysts investigated here with the exception of the non-electrochemically stabilized GNR/AuPdMoO and GNR/AuPd catalysts (Table S3). In addition, the average sum of the $\%$ contents of the $\mathrm{M}-\mathrm{O}$ and $\mathrm{M}-\mathrm{OH}$ peaks was $46 \%$ (Table S3); this indicated a significant contribution of metal oxides in the catalysts evaluated.

The C 1s HR-XPS spectra exhibited a peak that was deconvoluted in five peaks for the different catalysts synthesized here (Figure S12 and Table S3). The high \% content observed corresponded to the $\mathrm{C}=\mathrm{C}$ and $\mathrm{C}-\mathrm{C}$ deconvoluted peaks related to the GNR; on average, these peaks amounted to about $83 \%$ in the catalysts synthesized in this study (Table S3). We were unable to definitely identify the presence of $\mathrm{N}$ in the GNR structure (Figure S12). In general, the low signal 
to noise ratio observed for all HR-XPS spectra for the $\mathrm{Au}, \mathrm{Pd}$, and Mo is due to the very low amounts of the metals $(\leq 6.4 \mathrm{wt} . \%)$ and their wide spatial distribution present in the catalysts. With the here employed catalyst loadings, the XPS measurements/results were close to the instrumental limit of common spectrometers. As an example, the estimated detection limit for Mo in the carbon matrix is $\sim 0.01$ at. $\%{ }^{2}$.

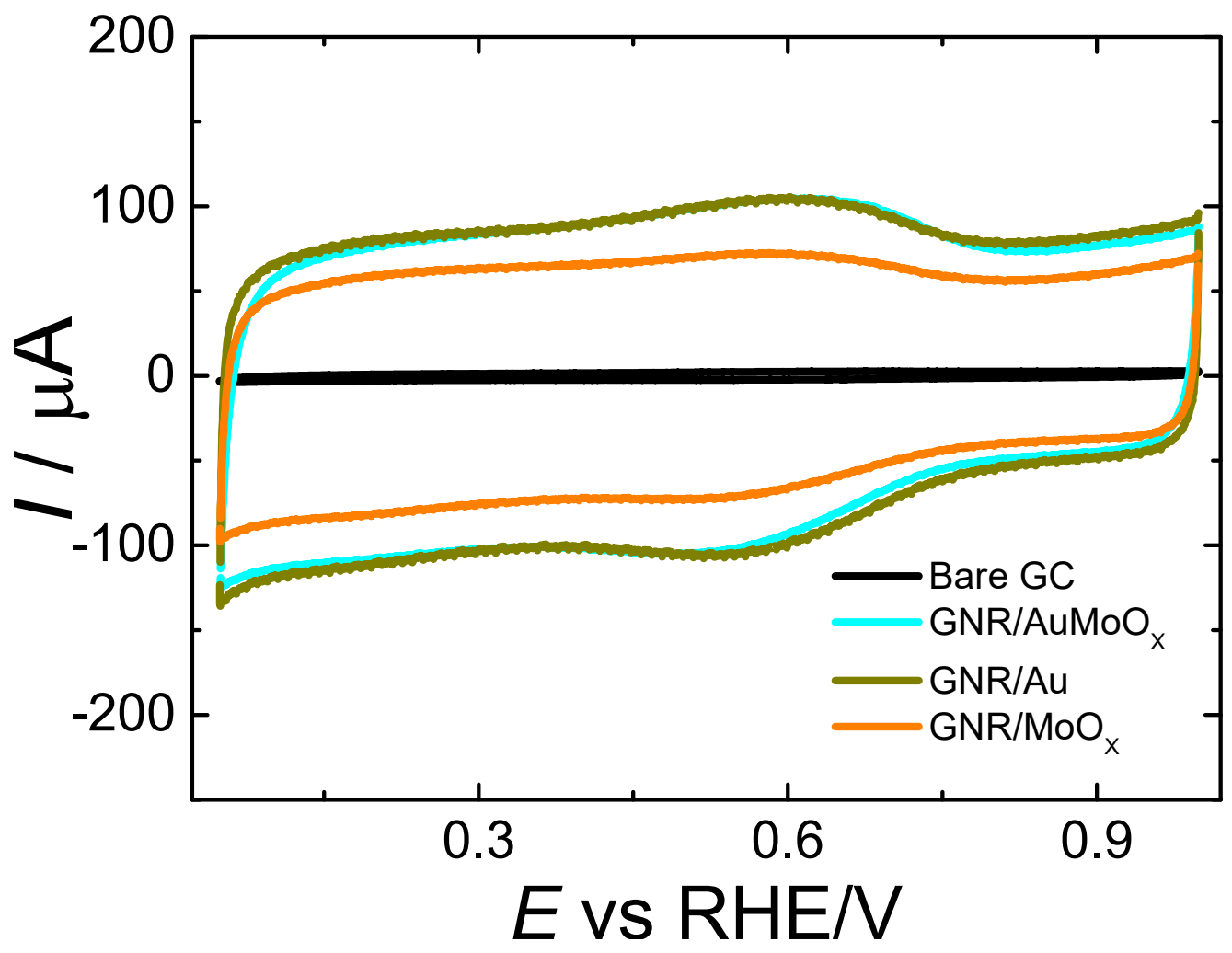

Figure S13.Cyclic voltammograms obtained for bare GC and GC electrodes modified with GNR/Metals catalysts applied in $\mathrm{N}_{2}$-saturated $0.1 \mathrm{M} \mathrm{HClO}_{4}$ using potential range of $0.05-1.0 \mathrm{~V}$ and scan rate of $50 \mathrm{mV} \mathrm{s}^{-1}$, starting at $1.0 \mathrm{~V}$. 

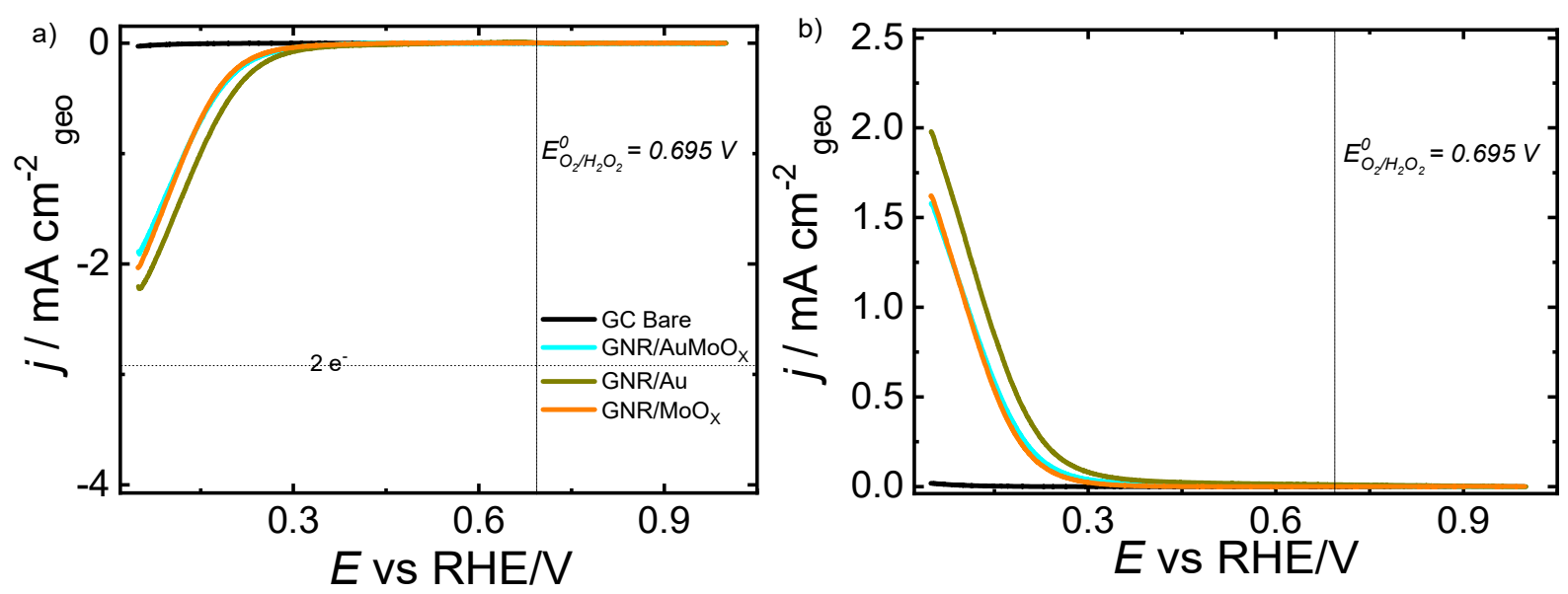

Figure S14. Results obtained from the linear sweep analysis conducted using RRDE for bare GC electrode and GC modified with GNR/AuMoO, $\mathrm{GNR} / \mathrm{Au}$ or $\mathrm{GNR} / \mathrm{MoO}_{\mathrm{x}}$ applied in $\mathrm{O}_{2}$-saturated $0.1 \mathrm{M} \mathrm{HClO}_{4}\left(\omega=1600 \mathrm{rpm}, v=10 \mathrm{mV} \mathrm{s}^{-1}\right)$ : (a) disk current densities. (b) ring current densities (curves normalized by $N$ - the collection efficiency $(0.26)$ ). 

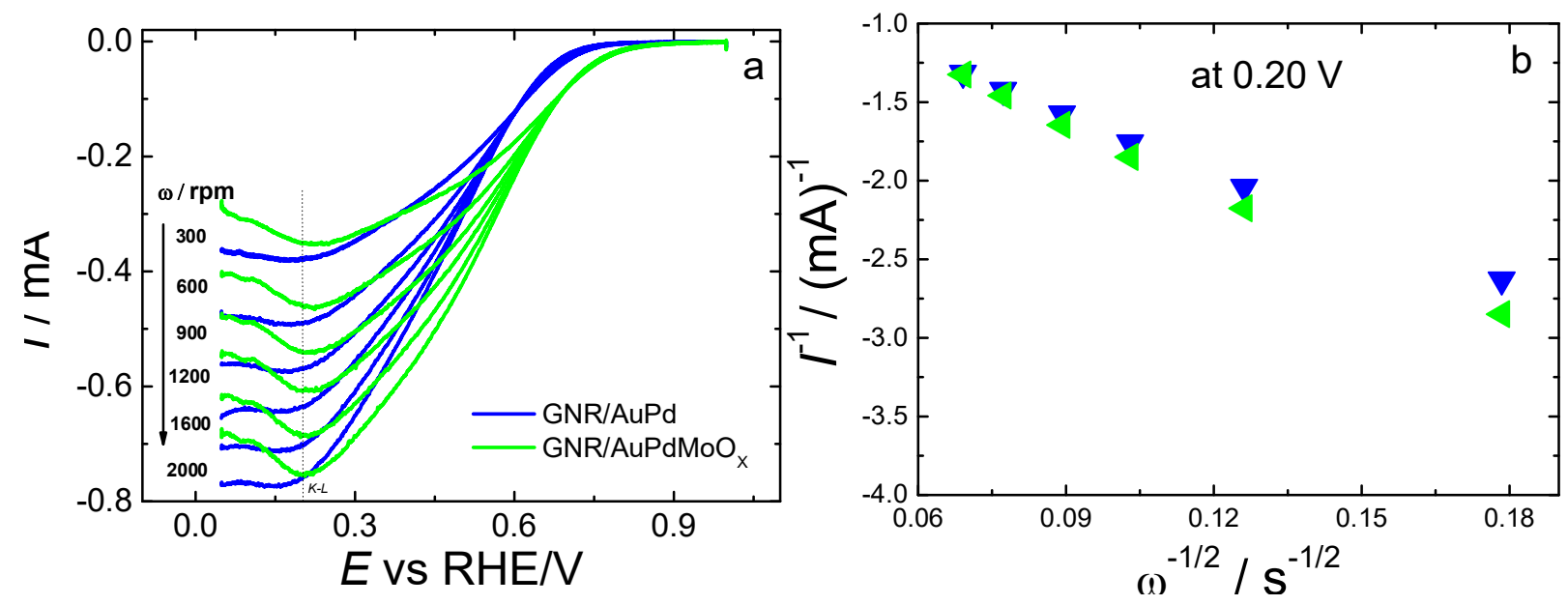

Figure S15. (a) Results obtained from the linear sweep analysis conducted using RRDE at various $\omega$ values for GC modified with GNR/AuPdMoO applied in $\mathrm{O}_{2}$-saturated $0.1 \mathrm{M} \mathrm{HClO}_{4}(v=10$ $\mathrm{mV} \mathrm{s}^{-1}$; scans started at $0.05 \mathrm{~V}$ ). (b) Koutecký-Levich plots (data from Figure a) were obtained using Equation S2. 


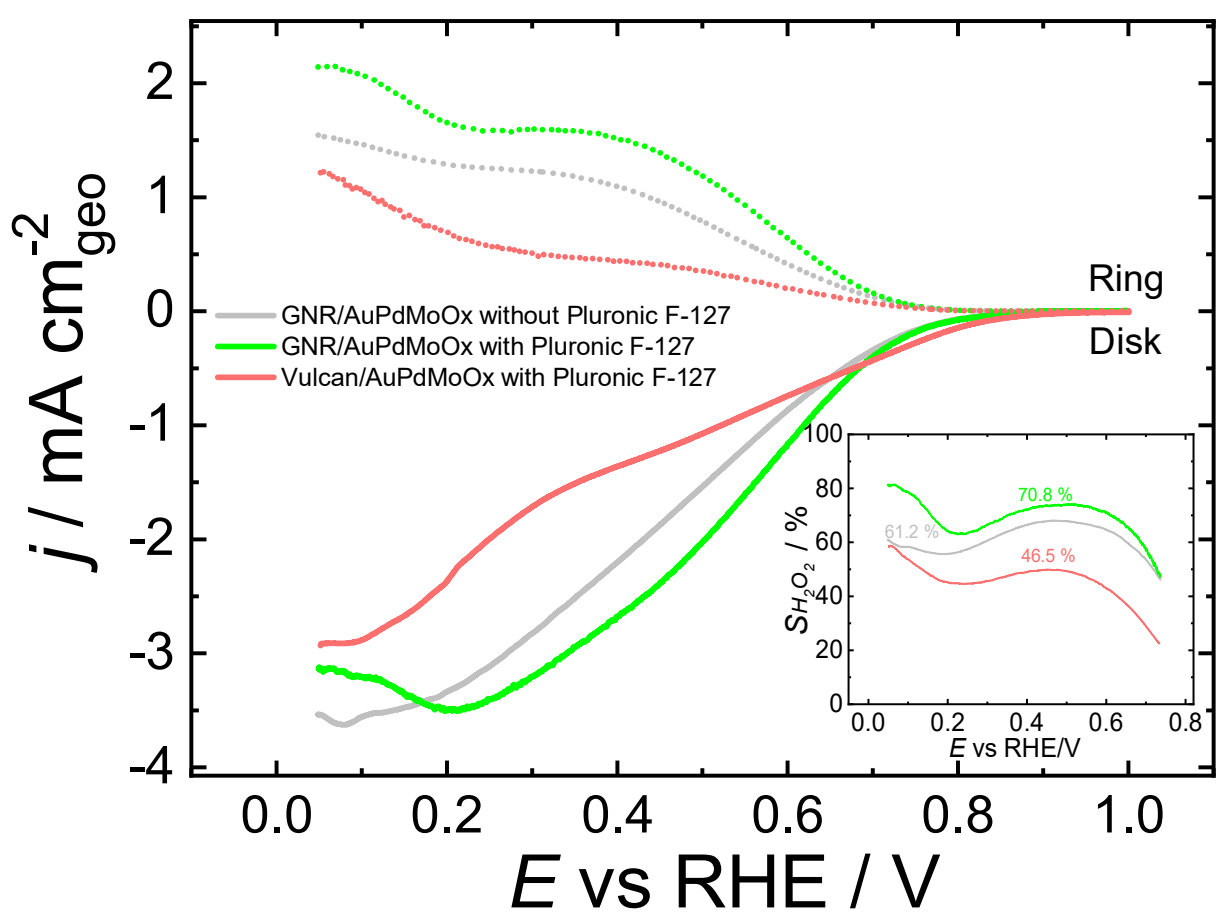

Figure S16. Results obtained from the linear sweep analysis conducted using RRDE for GC modified with GNR/AuPdMoO ${ }_{x}$ and Vulcan/AuPdMoO ${ }_{x}$ synthetized with or without the presence of the surfactant Pluronic F-127 applied in $\mathrm{O}_{2}$-saturated $0.1 \mathrm{M} \mathrm{HClO}_{4}(\omega=1600 \mathrm{rpm}, v=10 \mathrm{mV}$ $\mathrm{s}^{-1}$ ). Inset: selectivity for hydrogen peroxide production $\left(S_{\mathrm{H}_{2} \mathrm{O}_{2}}\right)$ during the ORR at varying potentials. 


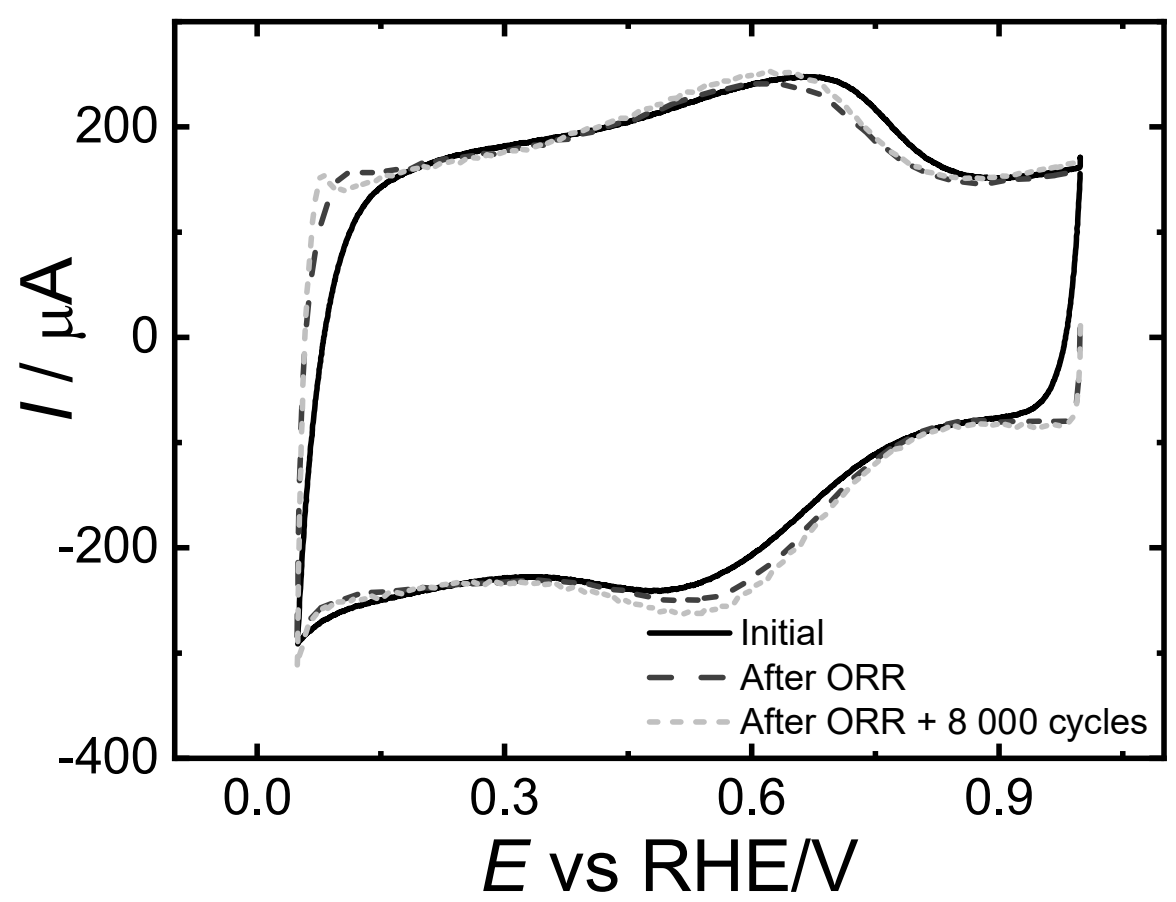

Figure S17. Cyclic voltammograms for non-electrochemically stabilized GC electrode modified with GNR/AuPdMoOx, after 3 consecutive ORR analyses (LSVs), and after the stability test, obtained in $\mathrm{N}_{2}$-saturated solution of $0.1 \mathrm{M} \mathrm{HClO}_{4}$. Potential scan rate: $50 \mathrm{mV} \mathrm{s}^{-1}$. Potential scan started at $1.0 \mathrm{~V} v s$. RHE. 


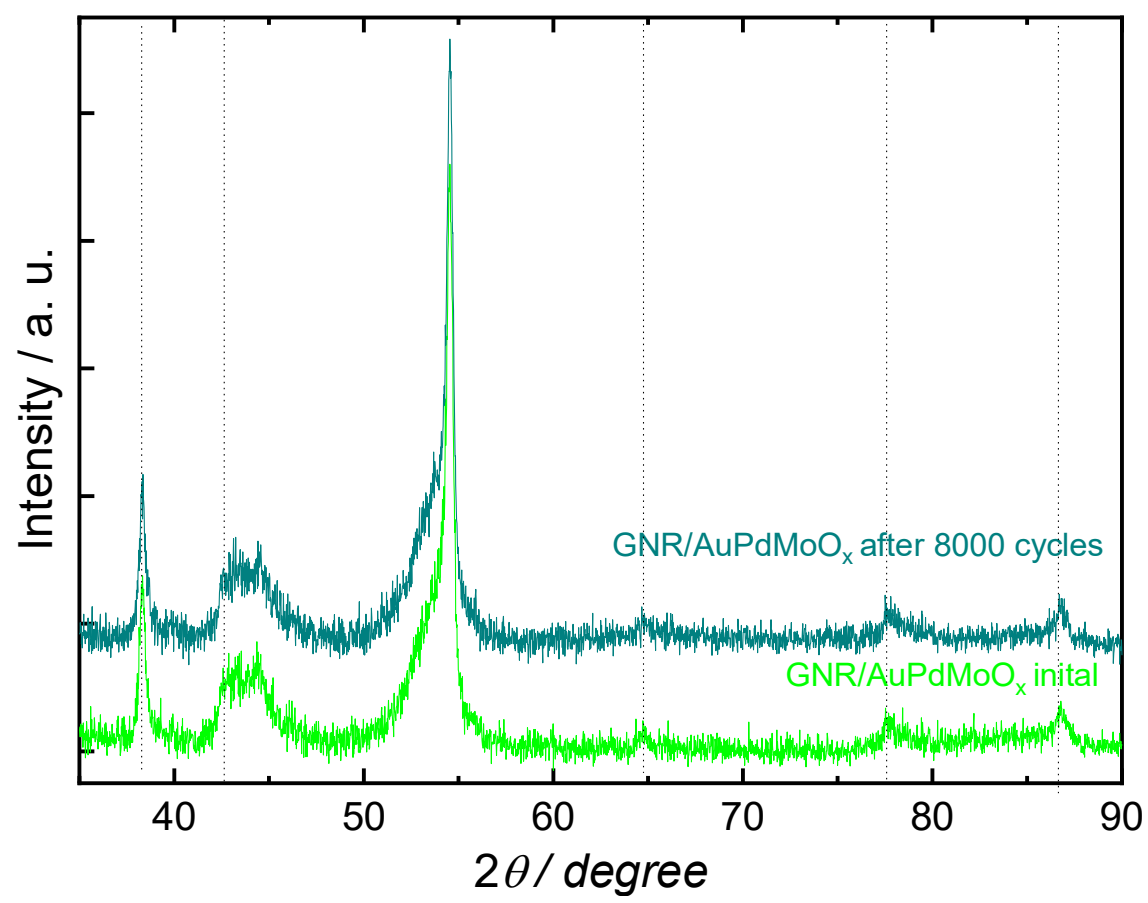

Figure S18. XRD patterns obtained for GNR/AuPdMoOx film supported on carbon paper (used as the working electrode) before and after the stability test. 


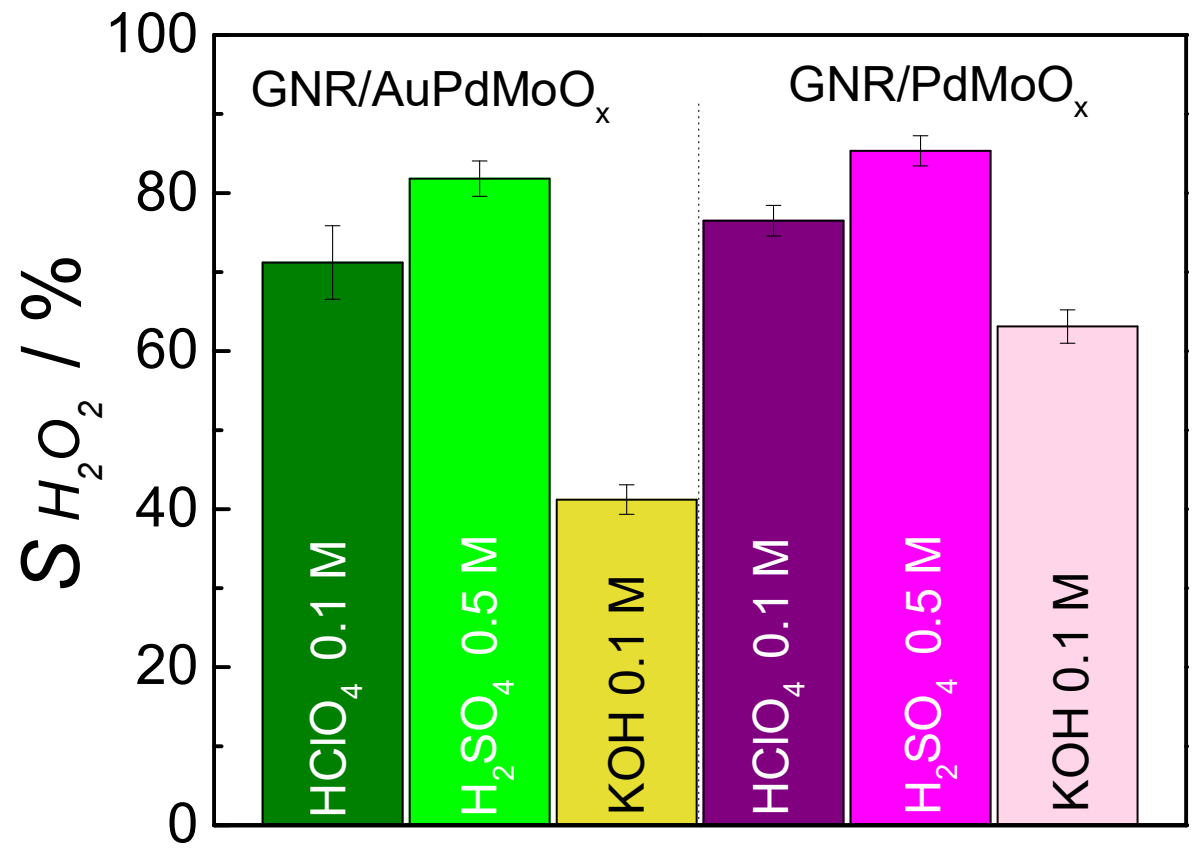

Figure S19. Average selectivity (\%) for $\mathrm{H}_{2} \mathrm{O}_{2}$ production over a potential range of $0.05-0.6 \mathrm{~V} v s$. RHE. Data obtained from Figure 8. 


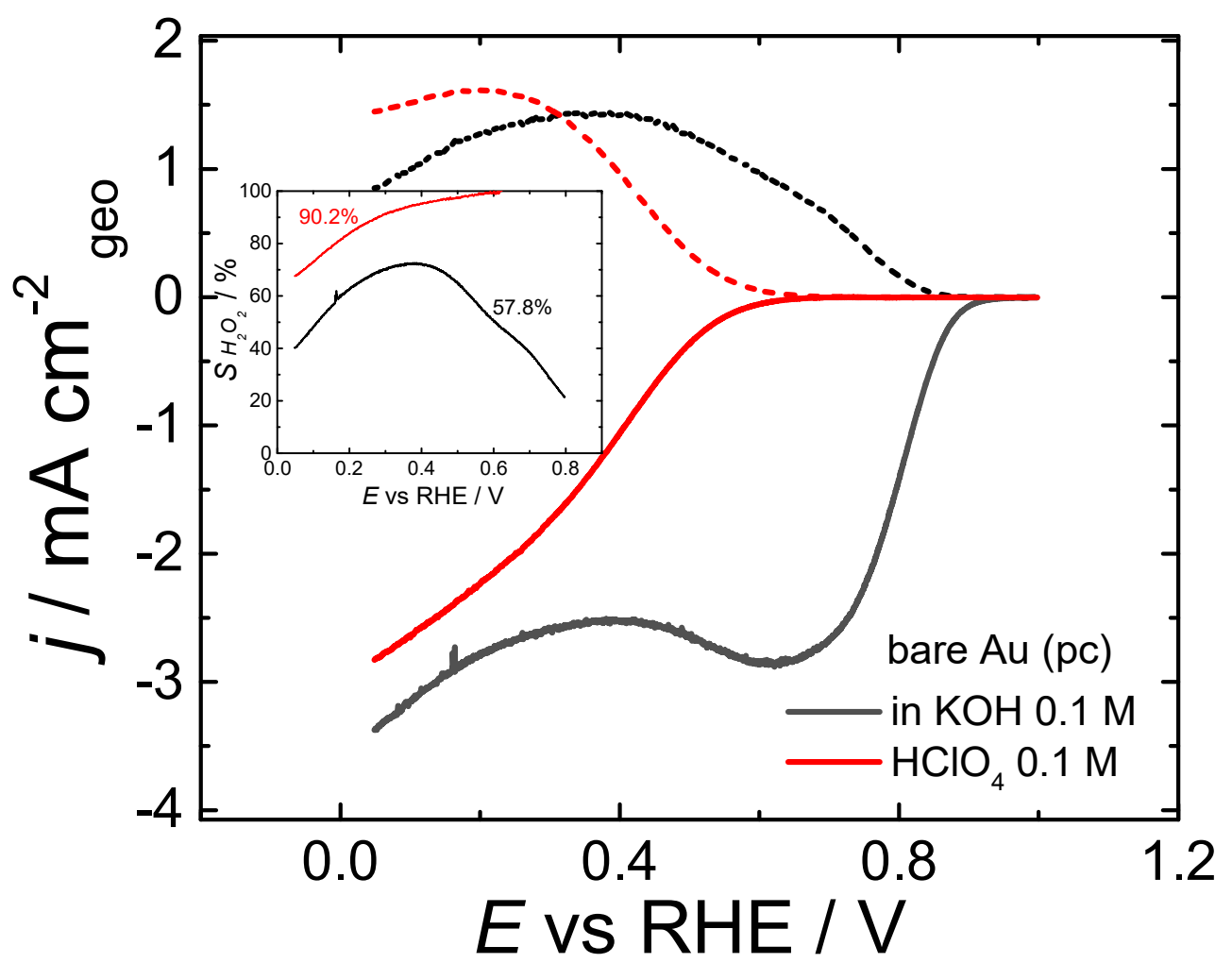

Figure S20. Results obtained from linear sweep analysis conducted using RRDE for polycrystalline (pc) Au bulk electrode applied in $\mathrm{O}_{2}$-saturated $0.1 \mathrm{M} \mathrm{HClO}_{4}(\omega=900 \mathrm{rpm}, v=10$ $\left.\mathrm{mV} \mathrm{s}^{-1}\right)$. Inset: selectivity for hydrogen peroxide production $\left(S_{\mathrm{H}_{2} \mathrm{O}_{2}}\right)$ during the ORR at varying potentials. The LSV curves were recorded using a RRDE electrode which consisted of a Teflonembedded Au disk/Pt ring rotating assembly $\left(0.196\right.$ and $0.11 \mathrm{~cm}^{2}$ in geometric area, respectively. Acquired from Pine Research Instrumentation). 
Table S6. Comparison table of ORR parameters for state-of-the-art catalysts toward $\mathrm{H}_{2} \mathrm{O}_{2}$ electroproduction measured by using a RRDE setup.

\begin{tabular}{|c|c|c|c|c|c|c|c|c|c|}
\hline Catalyst & $\begin{array}{l}\text { Total loading } \\
\quad(\mu \mathrm{g} \mathrm{cm}-2)\end{array}$ & $\begin{array}{l}\text { (Noble) metal } \\
\text { content }\end{array}$ & Electrolyte & 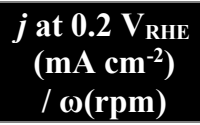 & $\begin{array}{c}E_{\text {onset }} \\
\left(V_{\text {RHE }}\right)\end{array}$ & $\begin{array}{c}\mathbf{E}_{1 / 2} \\
\left(\mathbf{V}_{\mathrm{RHE}}\right)\end{array}$ & $\begin{array}{c}\text { Selectivity } \\
(\%)\end{array}$ & $\begin{array}{c}\text { Mass activity (A } \\
\left.\text { mg }_{\text {metals }}^{-1}\right) / \eta \\
(\mathrm{mV})\end{array}$ & Ref \\
\hline GNR/AuPdMoOx & 153 & $\begin{array}{l}\text { Pd (1.2 wt\%) } \\
\text { Au }(4.0 \mathrm{wt} \%)\end{array}$ & $0.1 \mathrm{M} \mathrm{HClO}_{4}$ & $-3.5 / 1600$ & 0.82 & 0.54 & 71 & $0.07 / 50$ & This work \\
\hline GNR/AuPdMoOx & 153 & $\begin{array}{l}\text { Pd (1.2 wt\%) } \\
\text { Au (4.0 wt\%) }\end{array}$ & $0.5 \mathrm{M} \mathrm{H}_{2} \mathrm{SO}_{4}$ & $-2.3 / 1600$ & 0.77 & 0.53 & 82 & $0.05 / 50$ & This work \\
\hline GNR/PdMoOx & 153 & Pd (1.9 wt\%) & $0.1 \mathrm{M} \mathrm{HClO}_{4}$ & $-2.7 / 1600$ & 0.77 & 0.46 & 77 & $0.08 / 50$ & This work \\
\hline GNR/PdMoOx & 153 & Pd (1.9 wt\%) & $0.5 \mathrm{M} \mathrm{H}_{2} \mathrm{SO}_{4}$ & $-2.2 / 1600$ & 0.77 & 0.50 & 85 & $0.07 / 50$ & This work \\
\hline PdAu-nf & 100 & $\begin{array}{c}\mathrm{Pd}(1.8 \mathrm{wt} \%) \\
\mathrm{Au}(31.6 \mathrm{wt} \%)\end{array}$ & $0.1 \mathrm{M} \mathrm{HClO}_{4}$ & $-1.9 / 1600$ & 0.56 & 0.38 & $>90$ & - & Zhao et al. ${ }^{3}$ \\
\hline $\mathrm{Au}_{0.92} \mathrm{Pd}_{0.08} / \mathrm{C}$ & 110 & $\begin{array}{c}\mathrm{Pd}(0.71 \mathrm{wt} \%) \\
\mathrm{Au}(8.2 \mathrm{wt} \%)\end{array}$ & $0.1 \mathrm{M} \mathrm{HClO}_{4}$ & $-1.0 / 200$ & 0.59 & 0.32 & 95 & - & $\begin{array}{c}\text { Jirkovsky et } \\
\text { al. }^{4}\end{array}$ \\
\hline $\mathrm{PtHg} / \mathrm{C}$ & 25 & $\mathrm{Pt}(34 \mathrm{wt} \%)$ & $0.1 \mathrm{M} \mathrm{HClO}_{4}$ & $-0.8 / 1600$ & 0.80 & $\sim 0.42$ & $<90$ & $0.03 / 50$ & $\begin{array}{c}\text { Siahrostami et } \\
\text { al. }^{5}\end{array}$ \\
\hline $\mathrm{PdHg} / \mathrm{C}$ & 25 & $\mathrm{Pd}(53 \mathrm{wt} \%)$ & $0.1 \mathrm{M} \mathrm{HClO}_{4}$ & $-0.6 / 1600$ & 0.80 & $\sim 0.52$ & $>95$ & $0.13 / 50$ & $\begin{array}{c}\text { Verdaguer- } \\
\text { Casadevall et } \\
\text { al. }^{6}\end{array}$ \\
\hline $\mathrm{sc}-\mathrm{CoSe}_{2}$ & 200 & Co $(27 w t \%)$ & $0.5 \mathrm{M} \mathrm{H}_{2} \mathrm{SO}_{4}$ & $-1.2 / 400$ & $\sim 0.70$ & 0.35 & $>95$ & - & Zhang et al. $^{7}$ \\
\hline $\mathrm{C}(\mathrm{Pt}) / \mathrm{C}-4 \mathrm{~h}$ & 80 & $\mathrm{Pt}(60 \mathrm{wt} \%)$ & $1.0 \mathrm{M} \mathrm{HClO}_{4}$ & $-1.5 / 900$ & 0.71 & 0.41 & 41 & - & Choi et al. ${ }^{8}$ \\
\hline $\mathrm{h}-\mathrm{Pt}_{1}-\mathrm{CuS}_{\mathrm{x}} / \mathrm{C}$ & 100 & $\mathrm{Pt}(15 \mathrm{wt} \%)$ & $0.1 \mathrm{M} \mathrm{HClO}_{4}$ & $-2.8 / 1600$ & 0.67 & 0.42 & 96 & $0.07 / 250$ & Shen et al. ${ }^{9}$ \\
\hline $\mathrm{Pt}_{1} / \mathrm{TiC}$ & $\sim 100$ & $\mathrm{Pt}(0.2 \mathrm{wt} \%)$ & $0.1 \mathrm{M} \mathrm{HClO}_{4}$ & $-1.0 / 1600$ & 0.55 & 0.25 & 68 & $0.22 / 250$ & Yang et al. ${ }^{10}$ \\
\hline $\mathrm{Pt} / \mathrm{HSC}$ & 50 & $\operatorname{Pt}(5 \mathrm{wt} \%)$ & $0.1 \mathrm{M} \mathrm{HClO}_{4}$ & $-1.4 / 900$ & 0.60 & 0.35 & 95 & $0.10 / 250$ & Choi et al. ${ }^{11}$ \\
\hline $\mathrm{Pt} / \mathrm{TiN}$ & $\sim 100$ & $\mathrm{Pt}\left(0.35 \mathrm{wt}^{\%} \%\right)$ & $0.1 \mathrm{M} \mathrm{HClO}_{4}$ & $-2.5 / 1600$ & 0.50 & 0.30 & 65 & $0.08 / 50$ & Yang et al. ${ }^{12}$ \\
\hline $\mathrm{PdClx} / \mathrm{C}$ & 100 & $\operatorname{Pd}(1 \mathrm{wt} \%)$ & $0.1 \mathrm{M} \mathrm{HClO}_{4}$ & $-1.2 / 900$ & 0.69 & 0.38 & $>90$ & $0.07 / 50$ & $\begin{array}{l}\text { Ledendecker } \\
\text { et al. }{ }^{13}\end{array}$ \\
\hline $\mathrm{Pd}^{\delta+}-\mathrm{OCNT}$ & 50 & $\operatorname{Pd}(1 \mathrm{wt} \%)$ & $0.1 \mathrm{M} \mathrm{HClO}_{4}$ & $-2.0 / 1600$ & 0.70 & 0.31 & 98 & $1.95 / 200$ & Chang et al. ${ }^{14}$ \\
\hline GNR/PdMoOx & 150 & Pd (1.9 wt\%) & 0.1 М КОН & $-3.7 / 1600$ & 0.92 & 0.82 & 63 & $6.63 / 50$ & This work \\
\hline $\mathrm{Au}-\mathrm{Pt}-\mathrm{Ni} \mathrm{NRs}$ & 80 & $\begin{array}{c}\mathrm{Pt}+\mathrm{Au}(\sim 10 \\
\mathrm{wt} \%)\end{array}$ & $0.1 \mathrm{M} \mathrm{KOH}$ & $-2.0 / 1600$ & 0.70 & 0.57 & 95 & $0.19 / 150$ & Zheng et al. ${ }^{15}$ \\
\hline $\mathrm{FeC}-\mathrm{O}$ & 100 & $\mathrm{Fe}(0.1 \mathrm{at} \%)$ & $0.1 \mathrm{M} \mathrm{KOH}$ & $-3.2 * / 1600$ & 0.82 & 0.73 & $>95$ & $0.01 / 0 * *$ & Jiang et al. ${ }^{16}$ \\
\hline $\mathrm{Ni}-\mathrm{SA} / \mathrm{G}-0$ & 51 & $\mathrm{Ni}(1.5 \mathrm{wt} \%)$ & $0.1 \mathrm{M} \mathrm{KOH}$ & $-3.0 / 1600$ & 0.74 & 0.64 & $>94$ & $2.11 / 100$ & Song et al. ${ }^{17}$ \\
\hline O-CNTs & 100 & - & $0.1 \mathrm{M} \mathrm{KOH}$ & $-2.8 * / 1600$ & 0.79 & 0.70 & 90 & $<0.01 / 0 * *$ & Lu et al. ${ }^{18}$ \\
\hline
\end{tabular}




\section{Estimation of mass activity $(M A)$ toward $\mathrm{H}_{2} \mathrm{O}_{2}$ production}

The values related to $M A$ (in $\mathrm{A} \mathrm{g}^{-1}$ noble metal) obtained at an overpotential of $50 \mathrm{mV}$ (in relation to the thermodynamically expected value $-E_{\mathrm{O} 2 / \mathrm{H} 2 \mathrm{O} 2}^{0}=0.695 \mathrm{~V} v \mathrm{~s}$. RHE) were estimated according to the references ${ }^{1,5,19}$ and taking the following parameters into consideration: the kinetic current for $\mathrm{H}_{2} \mathrm{O}_{2}$ production ( $I_{\mathrm{k}}$, from ring curves), geometric $\mathrm{GC}$ surface area $\left(A_{\mathrm{g}}\right)$, and noble metal loading. The calculation was done based on Equation S1 below ${ }^{20,21}$.

$M A=\frac{I_{k}}{L_{\text {noble metal }} A_{g}}$

\section{Koutecký-Levich (K-L) plots}

K-L plots were constructed based on the data obtained from the analysis conducted using RRDE (Figs. S12 and S13; the calculation was done using Equation S2 below ${ }^{22}$ :

$\frac{1}{j}=\frac{1}{j k}+\frac{1}{j d}=-\frac{1}{n F k C_{O 2}^{b}}-\frac{1}{0.62 n F D_{O 2}^{2 / 3} v^{-1 / 6} C_{O 2}^{b} \omega^{1 / 2}}$

where $k$ is the rate constant for oxygen-reduction, $D_{O 2}$ is the $\mathrm{O}_{2}$ diffusion coefficient $\left(1.9 \times 10^{-5} \mathrm{~cm}^{2}\right.$ $\left.\mathrm{s}^{-1}\right)^{23}, v$ is the solution kinematic viscosity $\left(0.01 \mathrm{~cm} \mathrm{~s}^{-1}\right)^{24}, \mathrm{CO}_{O 2}{ }^{b}$ is the oxygen concentration in the solution $\left(1.2 \times 10^{-6} \mathrm{~mol} \mathrm{~cm}^{-3}\right)^{23}$, and $\omega$ is the rotation rate of the RRDE.

\section{REFERENCES}

(1) Fortunato, G. V.; Pizzutilo, E.; Mingers, A. M.; Kasian, O.; Cherevko, S.; Cardoso, E. S. F.; Mayrhofer, K. J. J.; Maia, G.; Ledendecker, M. Impact of Palladium Loading and Interparticle Distance on the Selectivity for the Oxygen Reduction Reaction toward Hydrogen Peroxide. J. Phys. Chem. C 2018, 122 (28), 15878-15885.

(2) Shard, A. G. Detection Limits in XPS for More than 6000 Binary Systems Using Al and Mg Ka X-Rays. Surf. Interface Anal. 2014, 46 (3), 175-185.

(3) Zhao, X.; Yang, H.; Xu, J.; Cheng, T.; Li, Y. Bimetallic PdAu Nanoframes for Electrochemical $\mathrm{H}_{2} \mathrm{O}_{2}$ Production in Acids. ACS Mater. Lett. 2021, 3 (7), 996-1002.

(4) Jirkovský, J. S.; Panas, I.; Ahlberg, E.; Halasa, M.; Romani, S.; Schiffrin, D. J. Single Atom Hot-Spots at Au-Pd Nanoalloys for Electrocatalytic $\mathrm{H}_{2} \mathrm{O}_{2}$ Production. J. Am. Chem. Soc. 2011, 133 (48), 19432-19441.

(5) Siahrostami, S.; Verdaguer-Casadevall, A.; Karamad, M.; Deiana, D.; Malacrida, P.; Wickman, B.; Escudero-Escribano, M.; Paoli, E. A.; Frydendal, R.; Hansen, T. W.; Chorkendorff, I.; Stephens, I. E. L.; Rossmeisl, J. Enabling Direct $\mathrm{H}_{2} \mathrm{O}_{2}$ Production through Rational Electrocatalyst Design. Nat. Mater. 2013, 12 (12), 1137-1143. 
(6) Verdaguer-casadevall, A.; Deiana, D.; Karamad, M.; Siahrostami, S.; Malacrida, P.; Hansen, T. W.; Rossmeisl, J.; Chorkendorff, I.; Stephens, I. E. L. Trends in the Electrochemical Synthesis of $\mathrm{H}_{2} \mathrm{O}_{2}$ : Enhancing Activity and Selectivity by Electrocatalytic Site Engineering. Nano Lett. 2014, 14 (3), 1603-1608.

(7) Zhang, X.; Su, X.; Zheng, Y.; Hu, S.; Shi, L.; Gao, F.; Yang, P.; Niu, Z.; Wu, Z.; Qin, S.; Wu, R.; Duan, Y.; Gu, C.; Zheng, X.; Zhu, J.; Gao, M. Strongly Coupled Cobalt Diselenide Monolayers for Selective Electrocatalytic Oxygen Reduction to $\mathrm{H}_{2} \mathrm{O}_{2}$ under Acidic Conditions. Angew. Chemie Int. Ed. 2021.

(8) Choi, C. H.; Kwon, H. C.; Yook, S.; Shin, H.; Kim, H.; Choi, M. Hydrogen Peroxide Synthesis via Enhanced Two-Electron Oxygen Reduction Pathway on Carbon-Coated Pt Surface. J. Phys. Chem. C 2014, 118 (51), 30063-30070.

(9) Shen, R.; Chen, W.; Peng, Q.; Lu, S.; Zheng, L.; Cao, X.; Wang, Y.; Zhu, W.; Zhang, J.; Zhuang, Z.; Chen, C.; Wang, D.; Li, Y. High-Concentration Single Atomic Pt Sites on Hollow CuSx for Selective $\mathrm{O}_{2}$ Reduction to $\mathrm{H}_{2} \mathrm{O}_{2}$ in Acid Solution. Chem 2019, 5 (8), 2099-2110.

(10) Yang, S.; Tak, Y. J.; Kim, J.; Soon, A.; Lee, H. Support Effects in Single-Atom Platinum Catalysts for Electrochemical Oxygen Reduction. ACS Catal. 2017, 7 (2), 1301-1307.

(11) Choi, C. H.; Kim, M.; Kwon, H. C.; Cho, S. J.; Yun, S.; Kim, H. H. T.; Mayrhofer, K. J. J. J.; Kim, H. H. T.; Choi, M. Tuning Selectivity of Electrochemical Reactions by Atomically Dispersed Platinum Catalyst. Nat. Commun. 2016, 7 (Iek 11), 1-9.

(12) Yang, S.; Kim, J.; Tak, Y. J.; Soon, A.; Lee, H. Single-Atom Catalyst of Platinum Supported on Titanium Nitride for Selective Electrochemical Reactions. Angew. Chemie Int. Ed. 2016, 55 (6), 2058-2062.

(13) Ledendecker, M.; Pizzutilo, E.; Malta, G.; Fortunato, G. V; Mayrhofer, K. J. J.; Hutchings, G. J.; Freakley, S. J. Isolated Pd Sites as Selective Catalysts for Electrochemical and Direct Hydrogen Peroxide Synthesis. ACS Catal. 2020, 5928-5938.

(14) Chang, Q.; Zhang, P.; Mostaghimi, A. H. B.; Zhao, X.; Denny, S. R.; Lee, J. H.; Gao, H.; Zhang, Y.; Xin, H. L.; Siahrostami, S.; Chen, J. G.; Chen, Z. Promoting $\mathrm{H}_{2} \mathrm{O}_{2}$ Production via 2-Electron Oxygen Reduction by Coordinating Partially Oxidized Pd with Defect Carbon. Nat. Commun. 2020, 11 (1), 2178.

(15) Zheng, Z.; Ng, Y. H.; Wang, D.-W.; Amal, R. Epitaxial Growth of Au-Pt-Ni Nanorods for Direct High Selectivity $\mathrm{H}_{2} \mathrm{O}_{2}$ Production. Adv. Mater. 2016, 28 (45), 9949-9955.

(16) Jiang, K.; Back, S.; Akey, A. J.; Xia, C.; Hu, Y.; Liang, W.; Schaak, D.; Stavitski, E.; Nørskov, J. K.; Siahrostami, S.; Wang, H. Highly Selective Oxygen Reduction to Hydrogen Peroxide on Transition Metal Single Atom Coordination. Nat. Commun. 2019, $10(1), 3997$.

(17) Song, X.; Li, N.; Zhang, H.; Wang, L.; Yan, Y.; Wang, H.; Wang, L.; Bian, Z. GrapheneSupported Single Nickel Atom Catalyst for Highly Selective and Efficient Hydrogen Peroxide Production. ACS Appl. Mater. Interfaces 2020, 12 (15), 17519-17527.

(18) Lu, Z.; Chen, G.; Siahrostami, S.; Chen, Z.; Liu, K.; Xie, J.; Liao, L.; Wu, T.; Lin, D.; Liu, Y.; Jaramillo, T. F.; Nørskov, J. K.; Cui, Y. High-Efficiency Oxygen Reduction to Hydrogen Peroxide Catalysed by Oxidized Carbon Materials. Nat. Catal. 2018, 1 (2), $156-162$. 
(19) Fortunato, G. V.; Pizzutilo, E.; Cardoso, E. S. F.; Lanza, M. R. V.; Katsounaros, I.; Freakley, S. J.; Mayrhofer, K. J. J.; Maia, G.; Ledendecker, M. The Oxygen Reduction Reaction on Palladium with Low Metal Loadings: The Effects of Chlorides on the Stability and Activity towards Hydrogen Peroxide. J. Catal. 2020, 389, 400-408.

(20) Paulus, U. A.; Schmidt, T. J.; Gasteiger, H. A.; Behm, R. J. Oxygen Reduction on a HighSurface Area Pt/Vulcan Carbon Catalyst: A Thin-Film Rotating Ring-Disk Electrode Study. J. Electroanal. Chem. 2001, 495 (2), 134-145.

(21) Antoine, O.; Durand, R. RRDE Study of Oxygen Reduction on Pt Nanoparticles inside Nafion ${ }^{\circledR}: \mathrm{H}_{2} \mathrm{O}_{2}$ Production in PEMFC Cathode Conditions. J. Appl. Electrochem. 2000, 30 (7), 839-844.

(22) Bard; Faulkner; Bard, A. J.; Faulkner, L. R. Electrochemical Methods: Fundamentals and Applications, 2nd ed.; John Wiley \& Sons: New York, 2001; Vol. 2.

(23) Davis, R. E.; Horvath, G. L.; Tobias, C. W. The Solubility and Diffusion Coefficient of Oxygen in Potassium Hydroxide Solutions. Electrochim. Acta 1967, 12 (3), 287-297.

(24) Lide, D. R. CRC Handbook of Chemistry and Physics, 84th Edition, 2003-2004; CRC Press: Boca Raton, 2001; Vol. 53. 